This page is not part of the technical paper.

10 April 2017

A note to all reviewers:

Active Vibration Damping (AVD) is a U.S. patented invention by two of the paper authors, as indicated in the paper reference. The technical details presented in this paper describing the implementation of AVD deliberately do not exceed the scope of the patent documentation which is already in the public domain. Also, there is insufficient information given in both this paper and the patent documentation for a technically minded person to implement AVD as described herein. Specifically, the derivation of the AVD angular rate controller algorithm from the AVD identified model is deliberately omitted. New information presented in this paper not in the public domain is the function of the AVD algorithm for the GOES-R series spacecraft and specifically the GOES-16 spacecraft.

Web link to patent documentation:

https://www.google.com/patents/US20080272240

Respectfully,

Brian Clapp

Lockheed Martin Space Systems Company 


\title{
GOES-R ACTIVE VIBRATION DAMPING CONTROLLER DESIGN, IMPLEMENTATION, AND ON-ORBIT PERFORMANCE
}

\author{
Brian R. Clapp ${ }^{(1)}$, Harald J. Weigl ${ }^{(2)}$, Neil E. Goodzeit ${ }^{(3)}$ \\ Delano R. Carter ${ }^{(4)}$, Timothy J. Rood ${ }^{(5)}$ \\ (1) Lockheed Martin Space Systems Company, P.O. Box 179, Denver CO 80201 \\ (303)971-4994, brian.clapp@lmco.com \\ (2) Lockheed Martin Space Systems Company, P.O. Box 179, Denver CO 80201 \\ harald.j.weigl@lmco.com \\ (3) Lockheed Martin Space Systems Company, P.O. Box 179, Denver CO 80201 \\ neil.e.goodzeit1@lmco.com \\ (4) Thearality, Inc., P.O. Box 12463, Glendale AZ 85318 \\ delano.r.carter@nasa.gov \\ (5) Advanced Solutions Inc., 7815 Shaffer Parkway, Littleton CO 80127 \\ timothy.j.rood@lmco.com
}

\begin{abstract}
GOES-R series spacecraft feature a number of flexible appendages with modal frequencies below $3.0 \mathrm{~Hz}$ which, if excited by spacecraft disturbances, can be sources of undesirable jitter perturbing spacecraft pointing. In order to meet GOES-R pointing stability requirements, the spacecraft flight software implements an Active Vibration Damping (AVD) rate control law which acts in parallel with the nadir point attitude control law. The AVD controller commands spacecraft reaction wheel actuators based upon Inertial Measurement Unit (IMU) inputs to provide additional damping for spacecraft structural modes below $3.0 \mathrm{~Hz}$ which vary with solar wing angle. A GOES-R spacecraft dynamics and attitude control system identified model is constructed from pseudo-random reaction wheel torque commands and IMU angular rate response measurements occurring over a single orbit during spacecraft post-deployment activities. The identified Fourier model is computed on the ground, uplinked to the spacecraft flight computer, and the AVD controller filter coefficients are periodically computed on-board from the Fourier model. Consequently, the AVD controller formulation is based not upon pre-launch simulation model estimates but upon on-orbit nadir point attitude control and timevarying spacecraft dynamics. GOES-R high-fidelity time domain simulation results herein demonstrate the accuracy of the AVD identified Fourier model relative to the pre-launch spacecraft dynamics and control truth model. The AVD controller on-board the GOES-16 spacecraft achieves more than a ten-fold increase in structural mode damping of the fundamental solar wing mode while maintaining controller stability margins and ensuring that the nadir point attitude control bandwidth does not fall below $0.02 \mathrm{~Hz}$. On-orbit GOES-16 spacecraft appendage modal frequencies and damping ratios are quantified based upon the AVD system identification, and the increase in modal damping provided by the AVD controller for each structural mode is presented. The GOES-16 spacecraft AVD controller frequency domain stability margins and nadir point attitude control bandwidth are presented along with on-orbit time domain disturbance response performance.
\end{abstract}




\section{INTRODUCTION}

The Geostationary Operational Environmental Satellite-R (GOES-R) spacecraft was launched on November 19, 2016, and the spacecraft is now on station in geosynchronous orbit and renamed GOES-16. The Lockheed Martin GOES-R series spacecraft are the next generation of advanced geosynchronous weather satellites for the United States operated by the National Oceanic and Atmospheric Administration (NOAA). When fully deployed, the GOES-R series spacecraft feature a number of flexible appendages with modal frequencies below $3.0 \mathrm{~Hz}$ which, if excited by spacecraft disturbances, can be sources of undesirable jitter perturbing spacecraft pointing. In order to meet the GOES-R Guidance Navigation and Control (GN\&C) subsystem pointing stability requirement of 221 $\mu \mathrm{rad}$ (or 46 arcseconds) peak-to-peak per axis over 60 seconds, the GOES-R flight software implements an Active Vibration Damping (AVD) rate control law which acts in parallel with the nominal mission Single Input Single Output (SISO) nadir point attitude control law. The AVD controller commands spacecraft reaction wheel actuators based upon Inertial Measurement Unit (IMU) inputs to provide additional damping for spacecraft structural modes below $3.0 \mathrm{~Hz}$ which vary with solar wing angle.

A GOES-16 spacecraft dynamics and attitude control system identified model is constructed from pseudo-random reaction wheel torque commands and IMU angular rate response measurements occurring over a single orbit during spacecraft post-deployment activities. Ground processing algorithms perform a least-squares Fourier fit of these data as a function of solar wing angle to identify the exact character of the combined spacecraft on-orbit structural dynamics and nadir point attitude control law. The Fourier model coefficients are uplinked and stored on-board the spacecraft flight computer and used over the entire mission duration; at every two-degrees of solar wing rotation, the AVD controller filter coefficients are updated on-board from the identified Fourier model. Consequently, the AVD controller formulation is based not upon pre-launch simulation model estimates but upon on-orbit nadir point attitude control and time-varying spacecraft dynamics.

This document includes four main sections, numbered as Section 2 through Section 5. Section 2 presents an overview of the GOES-R series spacecraft deployed configuration which identifies the flexible appendage structures. Section 3 describes the three component parts of the AVD algorithm: on-orbit AVD excitation, ground-based AVD system identification, and on-orbit AVD compensation.

GOES-R series spacecraft high-fidelity time domain simulation results in Section 4 demonstrate the accuracy of the AVD identified Fourier model relative to the pre-launch spacecraft dynamics and control truth model. High-fidelity time domain simulation results also demonstrate the robust performance of the AVD controller in the presence of worst-case appendage modal parameter variations anticipated over the GOES-R spacecraft 15-year mission duration.

Section 5 presents extensive frequency and time domain on-orbit performance of the AVD controller for the GOES-16 spacecraft. The AVD identified Fourier model for the GOES-16 spacecraft is used to predict on-orbit disturbance rejection transfer functions, on-orbit modal parameters, on-orbit AVD controller stability margins, on-orbit nadir point attitude control bandwidth, and on-orbit impulse disturbance responses in the time domain. Finally, GOES-16 attitude error flight telemetry during multiple thruster firings demonstrates the on-orbit appendage vibration attenuation performance of the AVD controller. 


\section{esa}

GNC 2017 : 10th International ESA Conference on

Guidance, Nauigation \& Control Systems

29 May - 2 June 2017, Salzburg, Austria

\section{GOES-R SERIES SPACECRAFT CONFIGURATION}

The fully deployed GOES-R series spacecraft configuration is shown in Figure 1 and Figure 2 along with the spacecraft body axis coordinate frame. Figure 2 documents the positive solar wing rotation angle convention, the solar wing zero-angle position, and the sun-facing side of the solar array.

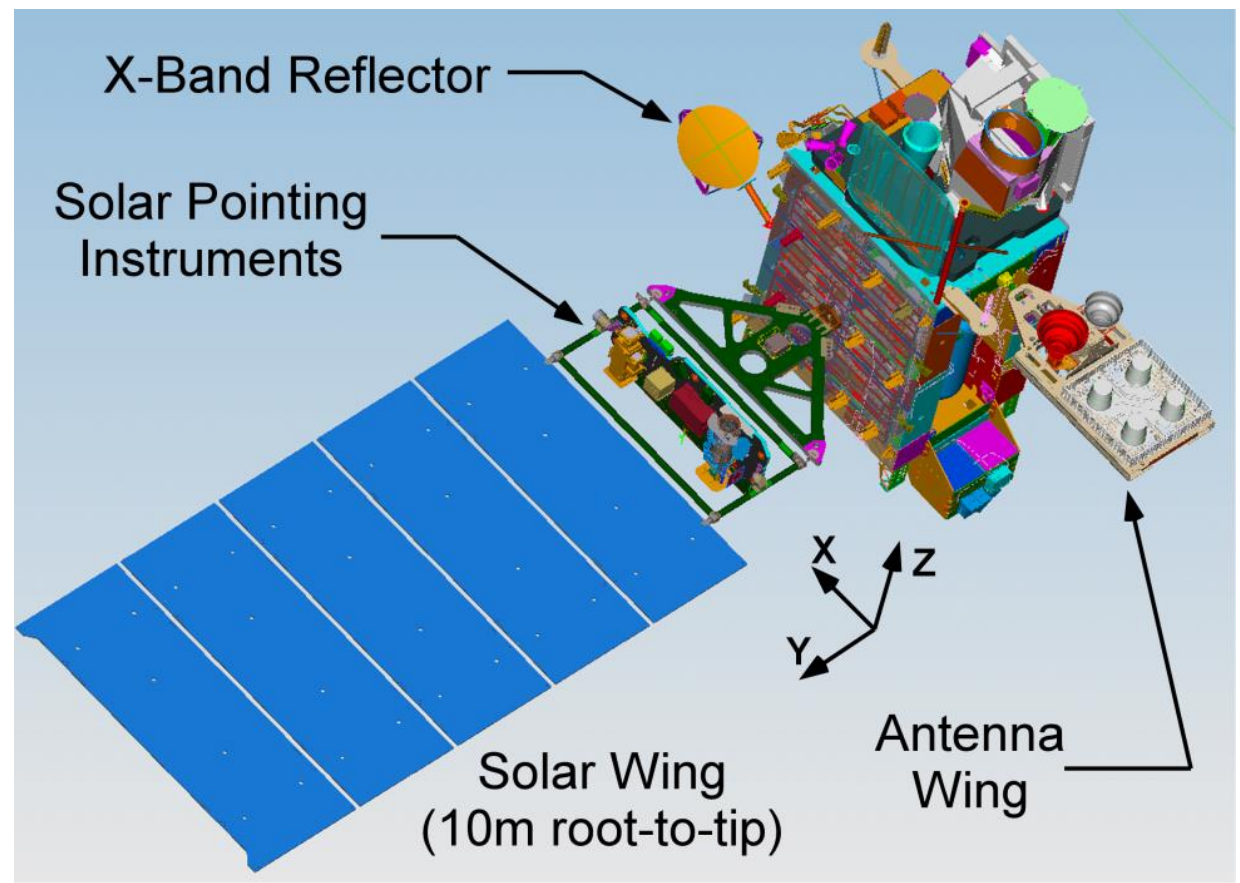

Figure 1. GOES-R Series Spacecraft in the Deployed Configuration

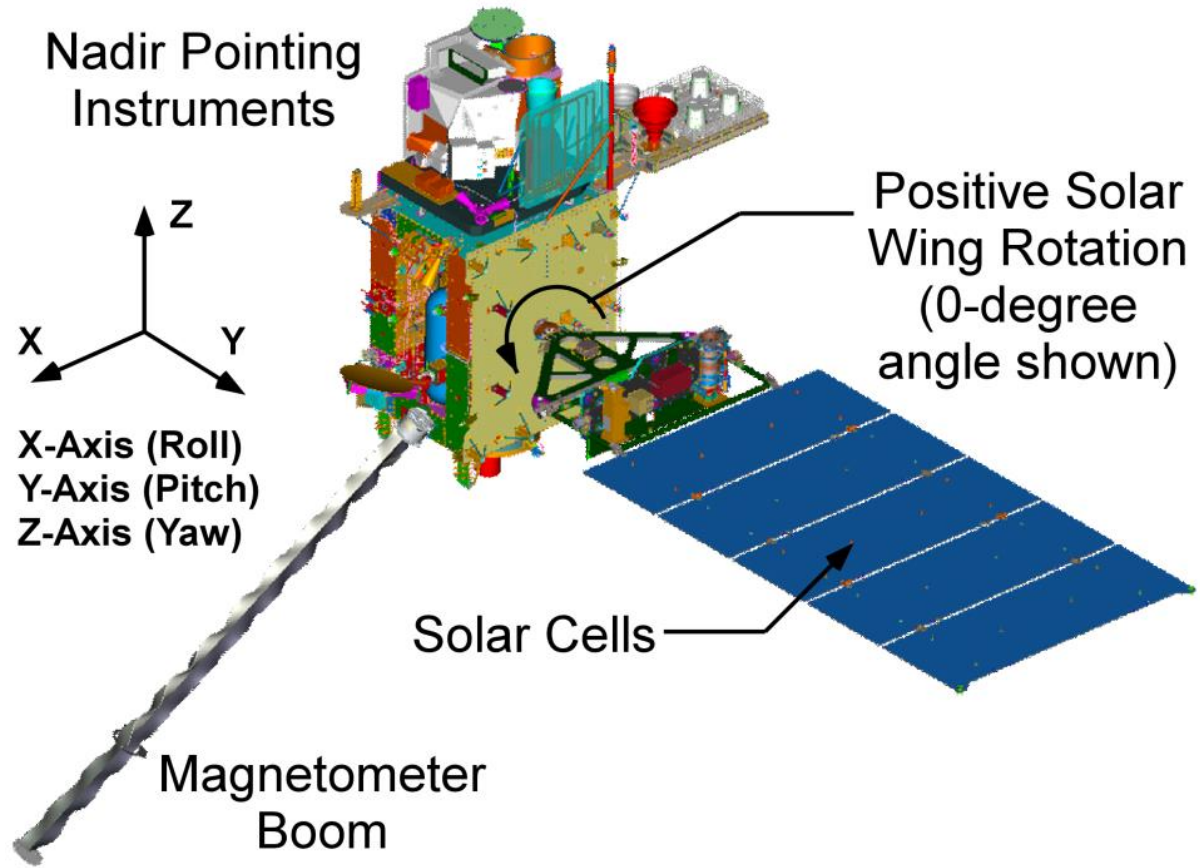

Figure 2. Deployed GOES-R Series Spacecraft and Solar Wing Angle Convention 
Each spacecraft carries a number of nadir pointing and solar pointing instruments as its primary payload. Each GOES-R series spacecraft features flexible appendages having bending modes below $3.0 \mathrm{~Hz}$ when fully deployed. The AVD system identification process identifies five solar wing natural modes, two magnetometer boom modes, and two antenna wing modes. The AVD identified Fourier model is used to quantify modal parameters for these nine flexible appendage modes, and the AVD controller provides additional damping to attenuate vibrations for the majority of these appendage natural modes.

\section{ACTIVE VIBRATION DAMPING ALGORITHM}

The AVD controller designed for the GOES-R series spacecraft is a system-identification based highbandwidth angular rate controller which acts in parallel with the attitude controller. AVD improves instrument pointing performance by applying phase stabilizing gain at the natural frequencies of the identified spacecraft appendage modes which modifies the spacecraft system dynamics, attenuates appendage vibration, and increases modal damping of the flexible appendages. AVD gain stabilizes all other spacecraft appendage modes with adequate margin to satisfy control system stability requirements. AVD also provides additional low-frequency disturbance attenuation control for all spacecraft axes which improves the performance of the nadir point attitude control law. AVD for the

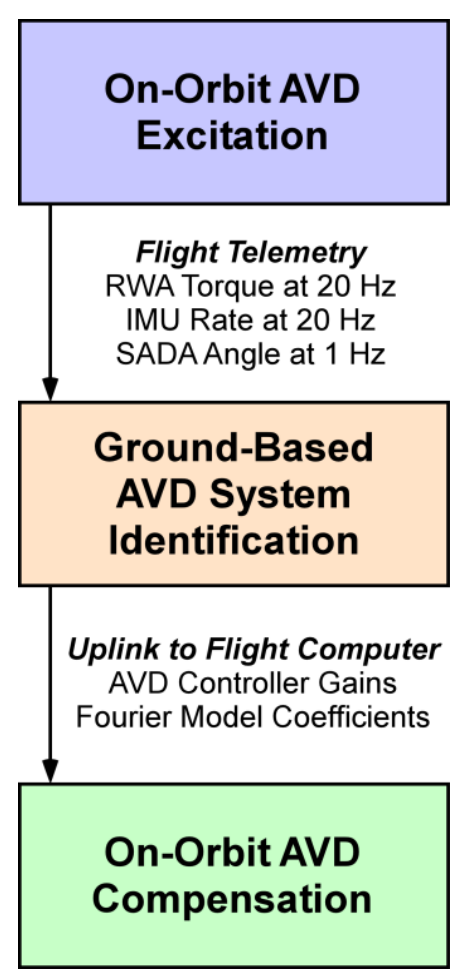

Figure 3. AVD Algorithm Components
GOES-R series spacecraft is implemented as a single-input singleoutput (SISO) rate controller used for each vehicle axis separately.

The AVD algorithm consists of three components which will be discussed separately in Section 3.1 through Section 3.3: a spacecraft flight software algorithm performing on-orbit AVD excitation, a ground-based AVD system identification process, and a spacecraft flight software algorithm performing on-orbit AVD compensation, as shown in Figure 3. GOES-R spacecraft flight telemetry from the AVD excitation process is passed to the ground-based AVD system identification process where the AVD identified Fourier model is computed as a function of solar wing angle. Additional ground processing is performed using the Fourier model to select the AVD controller gains. The AVD identified Fourier model coefficients and the AVD controller gains are subsequently uplinked to the spacecraft and stored in flight computer memory. The AVD compensation flight software algorithm periodically updates the AVD controller filter coefficients, which are computed from the identified Fourier model coefficients, the solar wing angle, and the AVD controller gains.

The AVD compensation algorithm, used on-board the spacecraft flight computer to compute the AVD controller filter coefficients, is used during ground processing to characterize all aspects of AVD controller performance after the identified Fourier model coefficients are computed. AVD controller disturbance rejection, stability margins, nadir point attitude control bandwidth, and time domain 
impulse responses are analyzed prior to uplinking the Fourier model coefficients and AVD controller gains to the spacecraft flight computer. Samples of these results for the GOES-16 spacecraft are documented in Section 5.

The AVD controller for the GOES-R series spacecraft is illustrated in the Figure 4 block diagram. When the AVD controller is disabled, the nominal nadir point controller provides attitude and rate control to stabilize spacecraft pointing. Upon enabling the AVD controller, the AVD compensation torque augments the nadir point attitude control torque, and both controllers provide combined attitude and rate control to stabilize the spacecraft and attenuate appendage vibration.

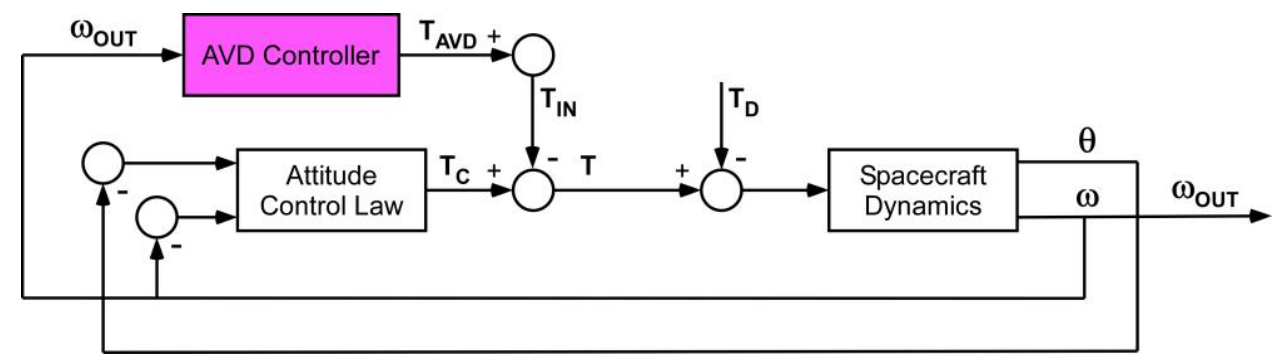

Figure 4. AVD Rate Controller and Closed-Loop Spacecraft Dynamics

\subsection{On-Orbit AVD Excitation Process}

GOES-R spacecraft dynamics and control are identified once during the spacecraft post-launch onorbit testing period and used over the entire mission duration. Because the GOES-R nadir point attitude control law is active during the system identification test, the closed-loop spacecraft dynamics and nadir point attitude control are identified together as a single Fourier model. To characterize the dependence of the spacecraft dynamics upon the solar wing angle, the system dynamics are identified over one complete solar wing rotation.

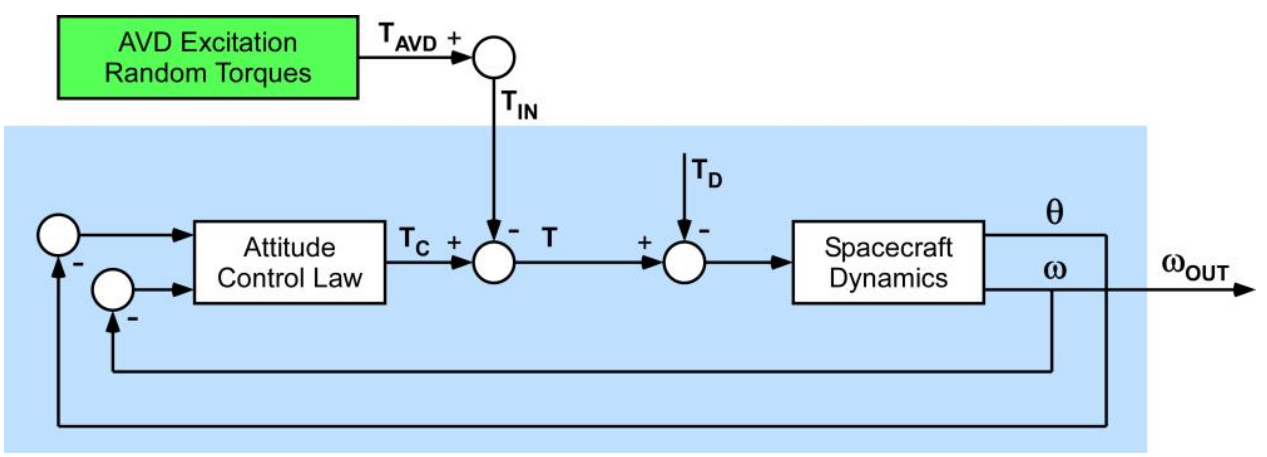

Figure 5. Spacecraft Configuration during AVD Excitation for System Identification

The spacecraft dynamics are excited by pseudo-random torques computed on-board and commanded to Reaction Wheel Assemblies (RWAs) during the on-orbit identification testing, as illustrated in Figure 5. AVD excitation is initiated by ground command. The response of the spacecraft to these inputs is determined by the IMU, which measures spacecraft body axis angular rates. The torque inputs and angular rate outputs telemetered at a $20 \mathrm{~Hz}$ sample rate are post-processed on the ground to identify the spacecraft dynamics as a function of solar wing angle. Identifying the spacecraft onorbit dynamics and control eliminates the dependence of the AVD controller performance on the accuracy of pre-flight system dynamic models because the dynamics of the spacecraft, sensors, 
actuators, and control processing are measured directly. The AVD identified model is represented by the shaded portion of Figure 5, which describes the torque input, $T_{I N}$, to angular rate output, $\omega_{\text {OUT }}$, system model for each spacecraft body axis.

AVD excitation is performed for each spacecraft axis separately every $10^{\circ}$ of solar wing rotation, and each excitation interval lasts five minutes. The torque excitation zero-to-peak magnitude used for GOES-16 was $0.9 \mathrm{Nm}$ per spacecraft body axis, and a representative angular rate response for the GOES-16 roll axis at a $90.5^{\circ}$ solar wing angle is shown in Figure 6. AVD excitation telemetry used for system identification consists of 36 torque input and 36 angular rate output time records per spacecraft body axis.
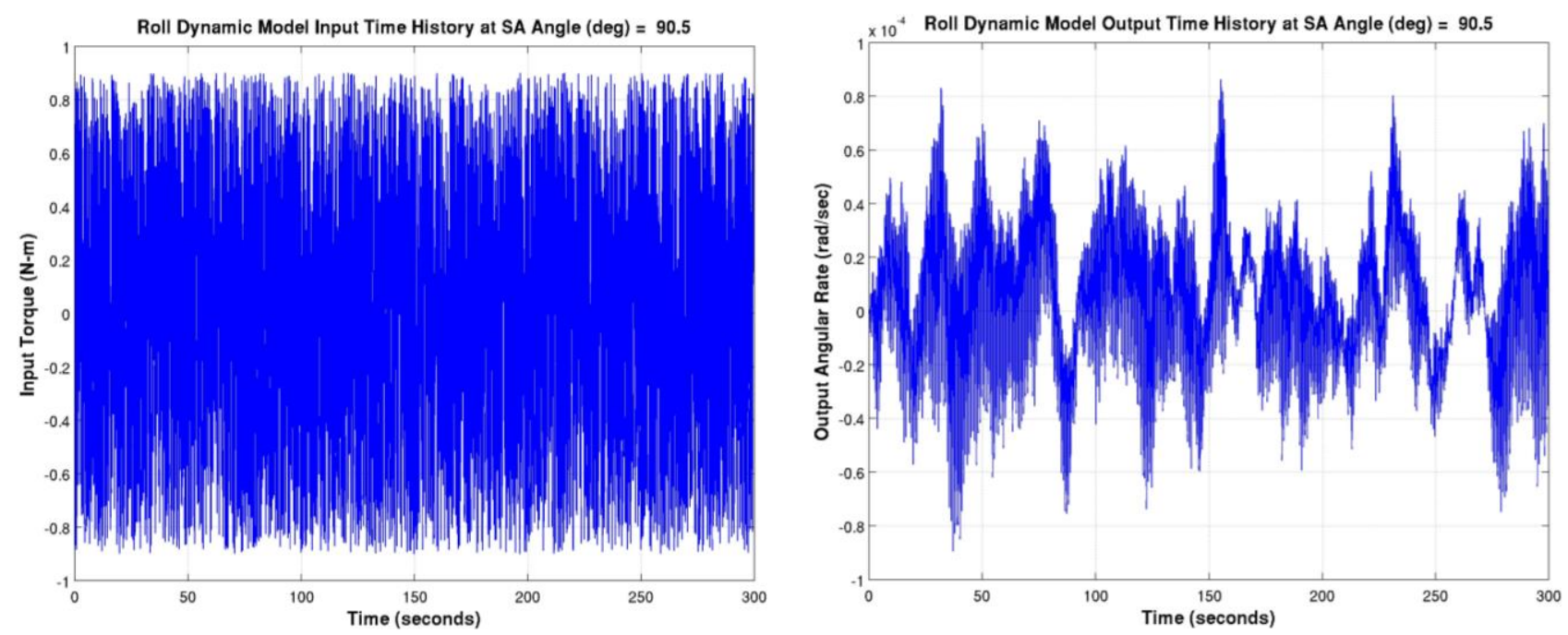

Figure 6. AVD Excitation Torque Input and Angular Rate Response Output for GOES-16

\subsection{Ground-Based AVD System Identification Process}

The measured spacecraft body rates, corresponding excitation torques, and solar wing angle are telemetered to the ground during post-launch testing of the spacecraft. This data is processed to estimate SISO transfer functions from RWA torque command to IMU angular rate response for each spacecraft body axis. The input-output data is collected for one full orbit period in order to capture the variation of the spacecraft dynamics over a $360^{\circ}$ range of solar wing angles.

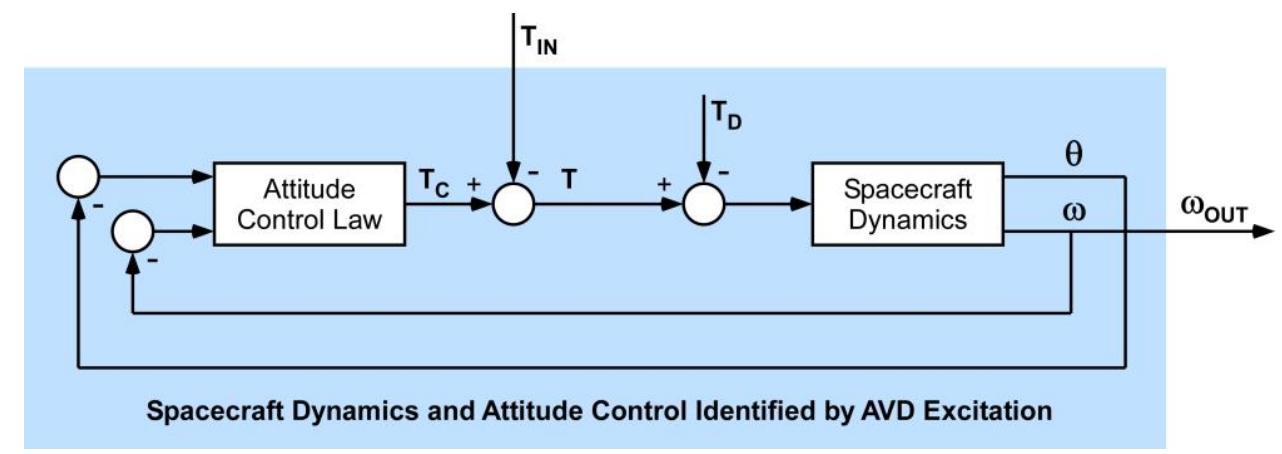

Figure 7. The AVD Identified Fourier Model 
The first objective of the system identification process is to use the telemetered spacecraft data to generate the AVD identified Fourier model illustrated in Figure 7. The identified model consists of three independent and uncoupled SISO system models, one for each spacecraft body axis, from spacecraft torque command input, $T_{I N}$, to angular rate response output, $\omega_{\text {OUT }}$. The shaded block diagram encompassing the closed-loop nadir point attitude control law and spacecraft system dynamics is used throughout this document to always indicate the AVD identified Fourier model generated by the system identification process described in this section.

The discrete-time AVD identified model transfer function, $G(z)$, for each spacecraft body axis is assumed to be of the form shown in Equation 1. It is a $p^{\text {th }}$ order RWA excitation torque, $T(z)$, to IMU angular rate response, $\omega(z)$, frequency domain transfer function. This is equivalent to the $p^{t h}$ order ARX (auto-regressive with exogenous input) discrete time domain model in Equation 2.

$$
\begin{gathered}
G(z)=\frac{\omega(z)}{T(z)}=\frac{b_{1} z^{-1}+b_{2} z^{-2}+\cdots+b_{p} z^{-p}}{1-a_{1} z^{-1}-a_{2} z^{-2}-\cdots-a_{p} z^{-p}} \\
\omega(k)=a_{1} \omega(k-1)+a_{2} \omega(k-2)+\cdots+a_{p} \omega(k-p)+b_{1} T(k-1)+b_{2} T(k-2)+\cdots+b_{p} T(k-p)
\end{gathered}
$$

The standard approach for identifying the parameters of an ARX model is extended in order to capture the dependence of spacecraft dynamics upon the solar wing angle, $\theta$. Equation 3 extends the ARX formulation of Equation 2 for the GOES-R series spacecraft to include periodicity of the identified model coefficients with respect to the solar wing angle. Each identified model coefficient in Equation 2 is represented by a ninth-order Fourier series in Equation 3. Sine and cosine terms follow the compact convention $s N \theta_{k}=\sin \left(N \theta_{k}\right)$ and $c N \theta_{k}=\cos \left(N \theta_{k}\right)$. The $k$ subscript of the solar wing angle, $\theta_{k}$, indicates the angular rate response at discrete time, $k$, also depends upon the solar wing angle at time $k$.

$$
\begin{aligned}
\omega(k) & =\left[A_{10}+A_{11} s \theta_{k}+A_{12} c \theta_{k}+A_{13} s 2 \theta_{k}+A_{14} c 2 \theta_{k}+A_{15} s 3 \theta_{k}+A_{16} c 3 \theta_{k}+A_{17} s 4 \theta_{k}+A_{18} c 4 \theta_{k}\right] \omega(k-1) \\
& +\left[A_{20}+A_{21} s \theta_{k}+A_{22} c \theta_{k}+A_{23} s 2 \theta_{k}+A_{24} c 2 \theta_{k}+A_{25} s 3 \theta_{k}+A_{26} c 3 \theta_{k}+A_{27} s 4 \theta_{k}+A_{28} c 4 \theta_{k}\right] \omega(k-2) \\
& +\cdots \\
& +\left[A_{p 0}+A_{p 1} s \theta_{k}+A_{p 2} c \theta_{k}+A_{p 3} s 2 \theta_{k}+A_{p 4} c 2 \theta_{k}+A_{p 5} s 3 \theta_{k}+A_{p 6} c 3 \theta_{k}+A_{p 7} s 4 \theta_{k}+A_{p 8} c 4 \theta_{k}\right] \omega(k-p) \\
& +\left[B_{10}+B_{11} s \theta_{k}+B_{12} c \theta_{k}+B_{13} s 2 \theta_{k}+B_{14} c 2 \theta_{k}+B_{15} s 3 \theta_{k}+B_{16} c 3 \theta_{k}+B_{17} s 4 \theta_{k}+B_{18} c 4 \theta_{k}\right] T(k-1) \\
& +\left[B_{20}+B_{21} s \theta_{k}+B_{22} c \theta_{k}+B_{23} s 2 \theta_{k}+B_{24} c 2 \theta_{k}+B_{25} s 3 \theta_{k}+B_{26} c 3 \theta_{k}+B_{27} s 4 \theta_{k}+B_{28} c 4 \theta_{k}\right] T(k-2) \\
& +\cdots \\
& +\left[B_{p 0}+B_{p 1} s \theta_{k}+B_{p 2} c \theta_{k}+B_{p 3} s 2 \theta_{k}+B_{p 4} c 2 \theta_{k}+B_{p 5} s 3 \theta_{k}+B_{p 6} c 3 \theta_{k}+B_{p 7} s 4 \theta_{k}+B_{p 8} c 4 \theta_{k}\right] T(k-p)
\end{aligned}
$$

Equation 3 is the formulation of the SISO discrete-time AVD identified Fourier model for each spacecraft body axis. The GOES-R series spacecraft use a $140^{\text {th }}$ order model, $p=140$, which yields $1260 A_{i j}$ and $1260 B_{i j}$ coefficients per spacecraft body axis. The total number of Fourier coefficients is 7560 encompassing all three spacecraft axes. As mentioned earlier, these coefficients are uplinked 
to the spacecraft flight computer and stored in flight computer memory. The Fourier coefficients are computed by a standard least-squares solution approach performed during ground processing. Equation 3 is re-organized into the form shown in Equation 4 in order to separate the Fourier coefficients from the time dependent terms. Present values of the angular rate response, $\omega(i)$, are ordered on the left side of the equation in column matrix $\boldsymbol{\Omega}$, and all past time history values from flight telemetry are multiplied by sine and cosine multiples of the solar wing angle, $\theta_{i}$, and grouped into the huge matrix, $\boldsymbol{\Theta}$. The least-squares solution of the AVD identified Fourier model coefficients, $\boldsymbol{\Phi}$, is performed according to Equation 5.

$$
\boldsymbol{\Omega}=\left[\begin{array}{c}
\vdots \\
\omega(i) \\
\vdots
\end{array}\right]=\boldsymbol{\Theta} \boldsymbol{\Phi}=\left[\begin{array}{c}
\omega(i-1) s \theta_{i} \omega(i-1) c \theta_{i} \omega(i-1) s 2 \theta_{i} \omega(i-1) c 2 \theta_{i} \omega(i-1) \ldots s 4 \theta_{i} T(i-p) c 4 \theta_{i} T(i-p) \\
\vdots
\end{array}\right]\left[\begin{array}{c}
A_{10} \\
A_{11} \\
A_{12} \\
A_{13} \\
A_{14} \\
A_{15} \\
A_{16} \\
A_{17} \\
A_{18} \\
\vdots \\
B_{p 0} \\
B_{p 1} \\
B_{p 2} \\
B_{p 3} \\
B_{p 4} \\
B_{p 5} \\
B_{p 6} \\
B_{p 7} \\
B_{p 8}
\end{array}\right]
$$

After computing all Fourier coefficients of the identified model in Equation 3, the practical use of the equation becomes clear. Given a particular GOES-R solar wing angle, $\theta$, the Fourier series coefficients in Equation 3 reduce to single $a_{i}$ and $b_{i}$ coefficients of Equation 1 and Equation 2, which can then be used for frequency domain or time domain analysis, respectively, of the identified system dynamics and control.

As mentioned earlier, the AVD controller filter coefficients are a function of the identified Fourier model coefficients, the solar wing angle, and the AVD controller gains. The development of the AVD control law formulation is proprietary technical information owned by Lockheed Martin and patented by two of the authors [1]. The algorithm to compute the AVD controller filter equations is implemented in the GOES-R series spacecraft flight computer. The algorithm computes the coefficients of the controller transfer function given by Equation 6, and the AVD control torque is computed using the discrete-time representation of Equation 7.

The SISO AVD controller is also a set of three $140^{\text {th }}$ order discrete transfer functions, one for each spacecraft body axis. The AVD controller coefficients $g_{i}$ and $h_{i}$ are a function of GOES-R solar wing angle, as they are computed directly from the AVD identified model coefficients $a_{i}$ and $b_{i}$ in Equation 2, which are, in turn, computed from Equation 3 given the solar wing angle of interest. The 
compensation provided by the AVD controller uniquely applies phase stabilizing gain at the natural frequencies of the identified spacecraft appendage modes to attenuate appendage vibration; all other spacecraft structural modes are gain stabilized.

$$
\begin{gathered}
H(z)=\frac{T(z)}{\omega(z)}=\frac{g_{1} z^{-1}+g_{2} z^{-2}+\cdots+g_{p} z^{-p}}{1-h_{1} z^{-1}-h_{2} z^{-2}-\cdots-h_{p} z^{-p}} \\
T(k)=h_{1} T(k-1)+h_{2} T(k-2)+\cdots+h_{p} T(k-p)+g_{1} \omega(k-1)+g_{2} \omega(k-2)+\cdots+g_{p} \omega(k-p)
\end{gathered}
$$

\subsection{On-Orbit AVD Compensation Process}

After completing system identification and verifying the performance and robustness of the AVD identification-based angular rate controller, the identified Fourier model is stored on-board the GOES-R series spacecraft flight computer. The identified model coefficients are used on-board to compute the AVD controller filter coefficients, which are updated every two degrees of solar wing rotation (every 8 minutes). The AVD controller receives IMU input at $20 \mathrm{~Hz}$ and computes $20 \mathrm{~Hz}$ control torque commands, which are summed with other feed-forward torque commands generated by the GOES-R attitude control system. AVD compensation is always active during nadir point operation of the GOES-R series spacecraft.

When the $140^{\text {th }}$ order AVD controller was first activated on-board the GOES-16 spacecraft on February 1, 2017, a perceptible 1.1\% increase in computational load was observed for the RAD750 flight computer processor. The computational load increase occurred because the AVD compensation torque per spacecraft body axis (Equation 7) is computed at $20 \mathrm{~Hz}$ from 140 past values of AVD torque and 140 past values of IMU angular rate measurements.

\section{AVD PRE-FLIGHT HIGH-FIDELITY SIMULATION FOR GOES-R}

The Lockheed Martin guidance and control team for the GOES-R program developed a high-fidelity spacecraft pointing control and jitter time domain simulation used to verify GOES-R spacecraft requirements during the design and development phase of the program. The high-fidelity simulation includes all aspects of the pointing control subsystem, and the simulation includes a detailed structural dynamic model of the GOES-R series spacecraft used to characterize the attitude control and structural dynamic interaction predicted for flight.

\subsection{AVD Identified Model Transfer Functions versus Spacecraft Dynamics Open-Loop Truth Model}

The on-orbit AVD identified Fourier model for GOES-R series spacecraft is one of the output products derived from the AVD system identification process. The identified model represents the true characteristics of the on-orbit spacecraft dynamics and control, but how accurately does the identified model predict the modal character of the spacecraft appendages? The AVD algorithm was implemented in the high-fidelity simulation to perform AVD excitation as described in Section 3.1, and the AVD ground processing system identification tools (Section 3.2) were used to post-process 
the simulated AVD excitation data to generate an AVD identified Fourier model. The identified model is compared with the open-loop system dynamic model from the high-fidelity simulation to demonstrate the ability of the AVD identified Fourier model to correctly characterize system appendage mode dynamics of the truth model. Examples of disturbance response transfer functions from body frame torque input to angular rate response output are shown in Figure 8 for the roll and yaw axes at a $0^{\circ}$ solar wing angle. As expected, the AVD identified model transfer functions nearly exhibit the characteristic $-20 \mathrm{~dB}$ per decade low-frequency roll-off associated with the attitude controller angular rate response, but the key characteristic illustrated in these figures is the ability of the AVD identified Fourier model to accurately predict the appendage modal peaks.
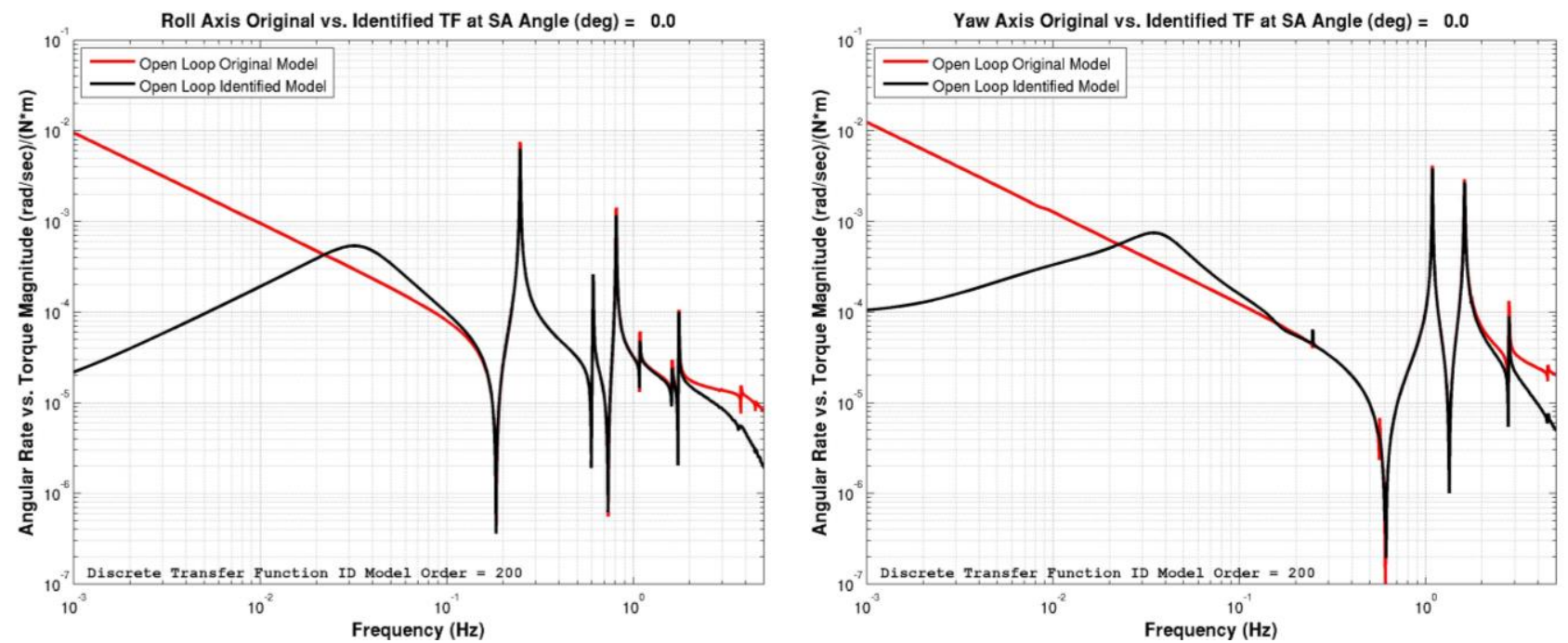

Figure 8. Open-Loop Spacecraft Dynamic Truth Model vs. AVD ID Model at $0^{\circ}$ SADA Angle

\subsection{AVD Identified Model Modal Parameters versus Spacecraft Dynamics Open-Loop Truth Model} Consistent with the disturbance response transfer functions presented in Section 4.1, the modal parameters are tabulated for nine appendage modes of the GOES-R series spacecraft. The modal parameters of the simulated AVD identified Fourier model are compared with the high-fidelity simulation open-loop system dynamics truth model.

Table 1: Modal Parameter Comparison Open-Loop Dynamics Truth vs. AVD ID Model

\begin{tabular}{|c|c|c|c|c|c|c|c|c|}
\hline \multirow[b]{2}{*}{ Appendage Flexible Mode Description } & \multirow[b]{2}{*}{$\begin{array}{l}\text { SADA } \\
\text { Angle } \\
\text { (deg) }\end{array}$} & \multirow[b]{2}{*}{$\begin{array}{l}\text { Body } \\
\text { Axis }\end{array}$} & \multicolumn{2}{|c|}{ Dynamics Truth Model } & \multicolumn{2}{|c|}{ AVD Identified Model } & \multicolumn{2}{|c|}{ Percent Difference } \\
\hline & & & $\begin{array}{c}\text { Frequency } \\
(\mathrm{Hz})\end{array}$ & $\begin{array}{l}\text { Damping } \\
\text { Ratio (\%) }\end{array}$ & $\begin{array}{l}\text { Frequency } \\
\text { (Hz) }\end{array}$ & $\begin{array}{l}\text { Damping } \\
\text { Ratio (\%) }\end{array}$ & Frequency & $\begin{array}{c}\text { Damping } \\
\text { Ratio }\end{array}$ \\
\hline Solar Wing Out-of-Plane Bending I & 0 & Roll & 0.247 & 0.200 & 0.247 & 0.237 & 0.0 & 18.3 \\
\hline Magnetometer Boom X-Rotation Bending & 330 & Roll & 0.611 & 0.200 & 0.611 & 0.208 & 0.0 & 4.0 \\
\hline Magnetometer Boom Y-Rotation Bending & 250 & Pitch & 0.564 & 0.200 & 0.563 & 0.206 & -0.2 & 3.2 \\
\hline Solar Wing Out-of-Plane Bending II & 0 & Roll & 0.808 & 0.200 & 0.807 & 0.238 & -0.1 & 19.2 \\
\hline Antenna Wing Out-of-Plane Bending & All & Pitch & 0.913 & 0.200 & 0.915 & 0.244 & 0.3 & 21.9 \\
\hline Solar Wing In-Plane Bending & 0 & Yaw & 1.083 & 0.200 & 1.083 & 0.211 & 0.0 & 5.3 \\
\hline Solar Wing Twist II & All & Pitch & 1.048 & 0.200 & 1.060 & 0.243 & 1.2 & 21.6 \\
\hline Solar Wing Out-of-Plane Bending III & 0 & Roll & 1.751 & 0.205 & 1.751 & 0.193 & 0.0 & -5.8 \\
\hline Antenna Wing In-Plane Bending & 0 & Yaw & 1.611 & 0.336 & 1.612 & 0.338 & 0.0 & 0.7 \\
\hline
\end{tabular}


Table 1 lists the modal frequencies and damping ratios for nine appendage modes of the high-fidelity simulation structural dynamics truth model and the AVD identified model. Modal parameter percentage differences are also tabulated. The AVD identified model determines the appendage modal frequencies within a $1.2 \%$ error; however, the identified model predicts modal damping within a $22 \%$ error. Because the AVD identified model is used in the AVD rate controller formulation to apply phase stabilizing gain at the spacecraft appendage modal frequencies, AVD controller effectiveness depends more upon accurate knowledge of appendage modal frequencies than damping ratios.

\subsection{AVD Controller Robustness Analysis}

Robustness of the AVD controller to appendage modal frequency variation was quantified using the GOES-R high-fidelity pointing control and jitter time domain simulation. Over the GOES-R series spacecraft 15 -year mission duration, no more than a $5 \%$ variation in appendage modal frequencies is anticipated due primarily to spacecraft mass and inertia changes as thruster propellant is depleted. Because the AVD identified Fourier model is derived once during post-launch testing and used over the entire mission duration, AVD controller effectiveness may decline as spacecraft appendage modal frequencies change over time.

Quantifying AVD controller robustness required generating 160 high-fidelity simulation structural dynamic models with uniformly varying appendage modal frequencies within $\pm 5 \%$ of the pre-launch nominal values. All simulations used the same AVD identified Fourier model to generate AVD controller filter coefficients based upon the nominal structural dynamic model. Time domain simulation scenarios evaluated GOES-R spacecraft solar instrument pointing errors, solar instrument pointing stability, nadir instrument peak attitude and angular rate errors, and nadir instrument pointing stability. Simulation study results demonstrated robust AVD controller performance in all cases, all simulation results remained compliant with all GOES-R pointing requirements, and spacecraft pointing performance was only slightly affected by appendage modal frequency variation with the nominal AVD controller. Even though the AVD excitation test is planned only once during postlaunch calibrations, AVD excitation calibration can be repeated at any time during the spacecraft mission to generate a new AVD identified Fourier model if spacecraft disturbance time responses begin to show unacceptable appendage vibration decay times affecting instrument pointing performance.

\section{AVD ON-ORBIT FLIGHT PERFORMANCE FOR GOES-16}

GOES-16 on-orbit AVD excitation was performed on 5-7 January 2017. Ground-based AVD system identification analysis commenced thereafter, and the AVD identified Fourier model coefficients and AVD controller gains were uplinked to the GOES-16 spacecraft on 1 February 2017. All frequency and time domain results presented in this section are based entirely upon the AVD identified Fourier model generated from GOES-16 AVD excitation flight telemetry. Section 5.1 through Section 5.5 present predicted on-orbit performance using the GOES-16 AVD identified model, whereas on-orbit AVD controller time domain performance from GOES-16 flight telemetry is presented in Section 5.6. These results demonstrate the spectacular on-orbit AVD controller performance for the GOES-16 spacecraft. 


\subsection{GOES-16 System-Identified Model Transfer Functions with AVD Disabled vs. AVD Enabled}

Disturbance torque input, $T_{I N}$, to angular rate response output, $\omega_{O U T}$, frequency domain transfer functions are generated for the GOES-16 AVD identified model based upon Figure 7; these are the GOES-16 on-orbit disturbance response transfer functions in nadir point control mode with the AVD controller disabled. For a particular solar wing angle, the GOES-16 AVD controller filter coefficients are computed by Equation (7) in Section 3.2, and the AVD controller loop is closed around the AVD identified model as shown in Figure 9. Disturbance torque input, $T_{C L}$, to angular rate response output, $\omega_{\text {OUT }}$, frequency domain transfer functions, computed using Figure 9, are the on-orbit disturbance responses of the GOES-16 spacecraft in nadir point control mode with the AVD controller enabled. Figure 10 and Figure 11 plot the GOES-16 disturbance rejection transfer functions which illustrate the increase in appendage modal damping with the AVD controller enabled as compared with the AVD controller disabled.

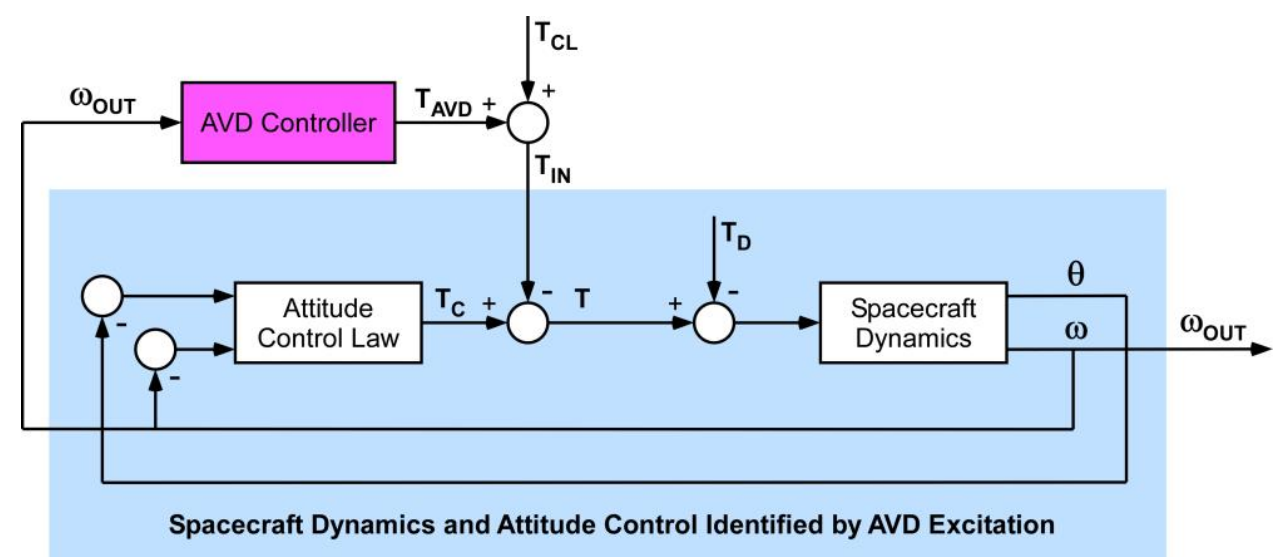

Figure 9. Closed-Loop AVD Controller with AVD Identified Model
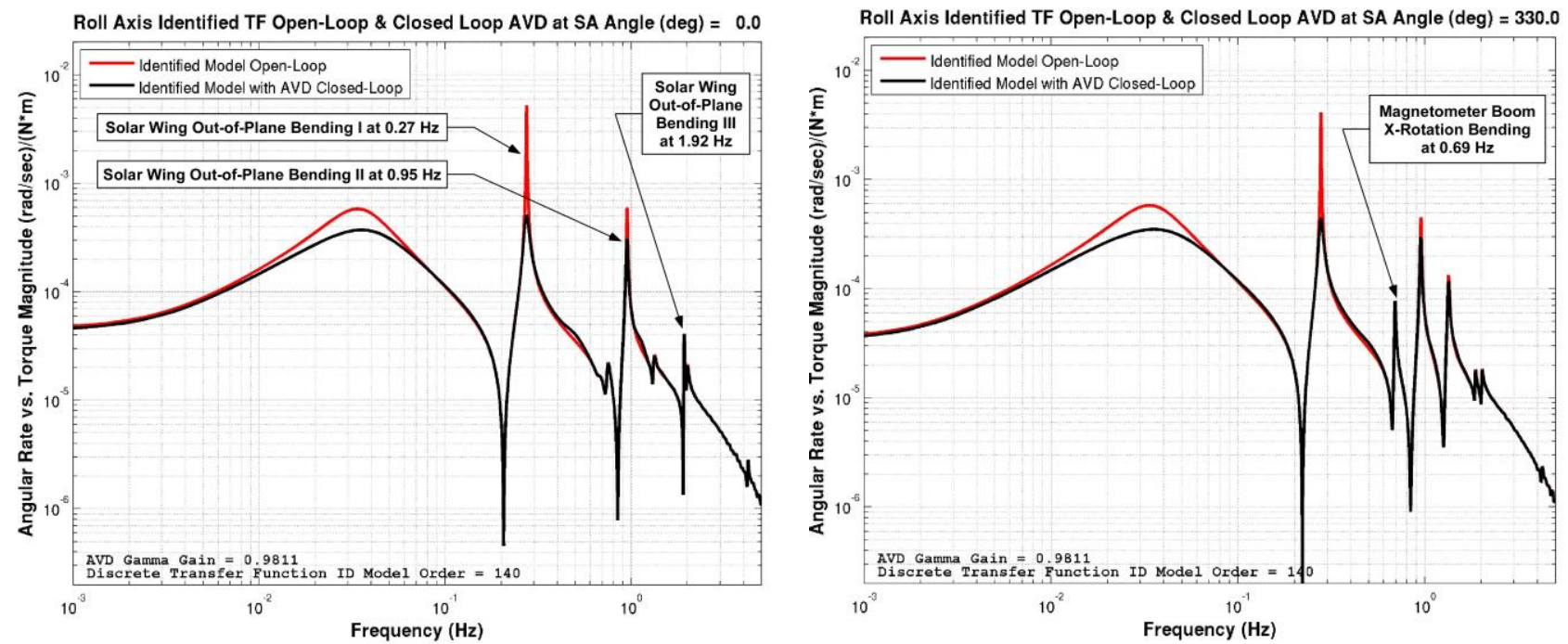

Figure 10. Roll Axis Disturbance Rejection with AVD Disabled vs. Enabled

Many of the GOES-16 disturbance torque input to angular rate output transfer functions generated using the AVD identified model do not show the ideal $-20 \mathrm{~dB}$ per decade low-frequency roll-off down to $0.001 \mathrm{~Hz}$, as illustrated in Figure 10 and Figure 11. Because 5-minute AVD excitation intervals 
are used for the GOES-16 AVD calibration, the minimum frequency content present in the AVD excitation telemetry data is $0.0033 \mathrm{~Hz}$. The low-frequency disturbance response characteristic is not a pointing performance concern because the AVD controller acts in parallel with the nadir point attitude control law, as shown in Figure 9, and the nadir point attitude controller attenuates very low frequency disturbances.
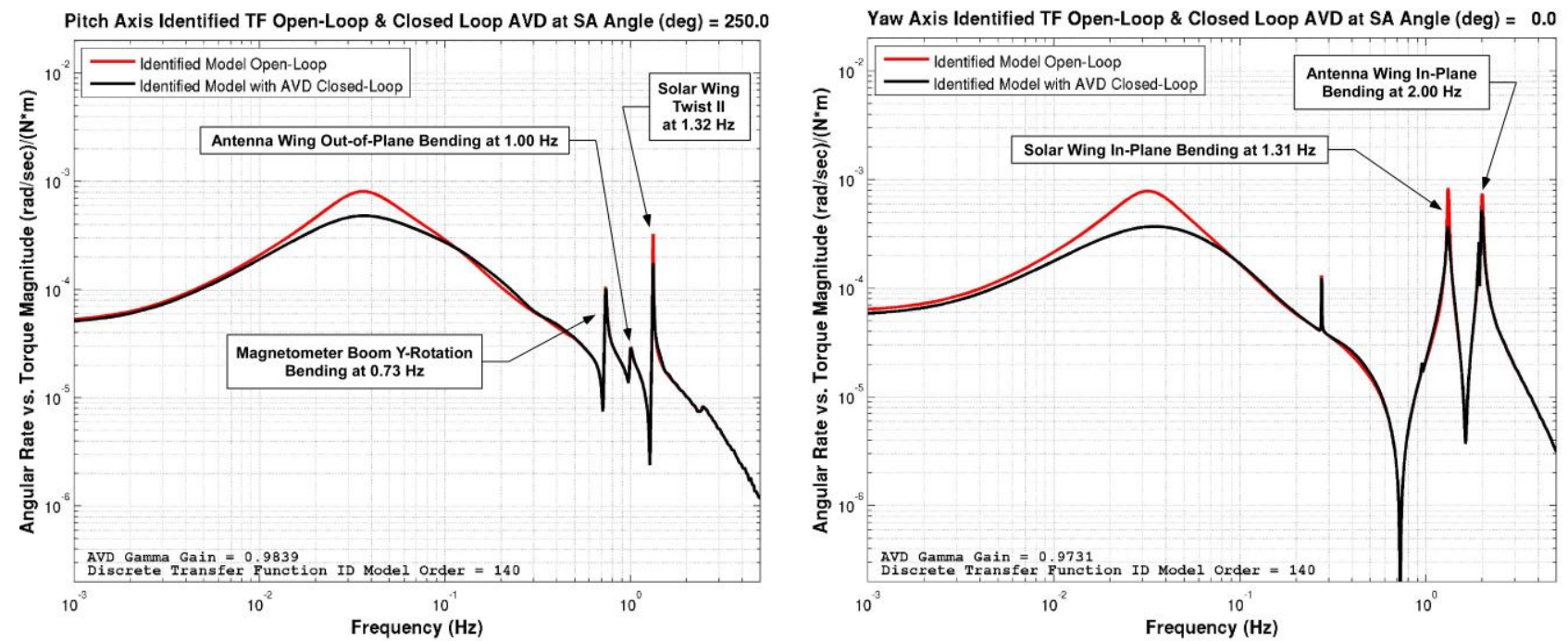

Figure 11. Pitch and Yaw Axis Disturbance Rejection with AVD Disabled vs. Enabled

\subsection{GOES-16 System-Identified Model Modal Parameters with AVD Disabled vs. AVD Enabled}

GOES-16 appendage modal parameters associated with the transfer functions shown in Figure 10 and Figure 11 are listed in Table 2. Modal parameters with the AVD controller disabled are the modal parameters of the AVD identified model. The modal parameters in Table 2 are representative of the AVD controller performance for the GOES-16 spacecraft for all solar wing angles.

Table 2: GOES-16 Modal Parameters AVD Disabled vs. AVD Enabled

\begin{tabular}{|l|c|c|c|c|c|c|}
\hline Appendage Flexible Mode Description & $\begin{array}{c}\text { SADA } \\
\text { Angle } \\
\text { (deg) }\end{array}$ & $\begin{array}{c}\text { Body } \\
\text { Axis }\end{array}$ & $\begin{array}{c}\text { Frequency } \\
\text { (Hz) }\end{array}$ & $\begin{array}{c}\text { Damping } \\
\text { Ratio (\%) } \\
\text { Identified }\end{array}$ & $\begin{array}{c}\text { Damping } \\
\text { Ratio (\%) } \\
\text { with AVD }\end{array}$ & $\begin{array}{c}\text { AVD } \\
\text { Damping } \\
\text { Factor }\end{array}$ \\
\hline \hline Solar Wing Out-of-Plane Bending I & 0 & Roll & 0.273 & 0.253 & 2.743 & 10.82 \\
\hline Magnetometer Boom X-Rotation Bending & 330 & Roll & 0.691 & 0.674 & 0.628 & 0.93 \\
\hline Magnetometer Boom Y-Rotation Bending & 250 & Pitch & 0.733 & 0.820 & 0.826 & 1.01 \\
\hline Solar Wing Out-of-Plane Bending II & 0 & Roll & 0.948 & 0.460 & 0.878 & 1.91 \\
\hline Antenna Wing Out-of-Plane Bending & All & Pitch & 0.999 & 7.191 & 7.182 & 1.00 \\
\hline Solar Wing In-Plane Bending & 0 & Yaw & 1.313 & 0.978 & 1.971 & 2.02 \\
\hline Solar Wing Twist II & All & Pitch & 1.320 & 0.279 & 0.366 & 1.31 \\
\hline Solar Wing Out-of-Plane Bending III & 0 & Roll & 1.923 & 0.321 & 0.305 & 0.95 \\
\hline Antenna Wing In-Plane Bending & 0 & Yaw & 1.996 & 0.840 & 1.149 & 1.37 \\
\hline
\end{tabular}

Note: AVD Damping Factor $=($ Modal Damping AVD Enabled $) /($ Modal Damping AVD Disabled $)$

The AVD controller does not increase modal damping for all appendage modes, but five of the nine appendage structural modes identified by AVD excitation realize an increase in modal damping. In particular, the AVD controller increases the modal damping for the GOES-16 fundamental solar wing 
mode (the first out-of-plane bending mode of the solar wing) by more than a factor of 10 . This is the benefit provided by AVD; the AVD controller is a flight software algorithm effectively adding damping to GOES-16 appendage hardware.

On-orbit GOES-16 appendage modal frequencies identified by AVD excitation are listed in Table 2. These appendage modal frequencies are higher than the pre-launch model-predicted appendage frequencies for GOES-R series spacecraft listed in Table 1. The lower model-predicted frequencies are due to conservatism built-in to structural dynamic finite element models developed during the GOES-R spacecraft design phase. This comparison illustrates a key benefit of the AVD controller formulation based upon on-orbit system identification dynamics and control models rather than less accurate pre-launch simulation models.

\subsection{GOES-16 Frequency Domain AVD Controller Stability Margins}

GOES-16 AVD controller stability margins are determined from the open-loop transfer function of the AVD controller in series with the AVD identified Fourier model, as illustrated by the block diagram in Figure 12.

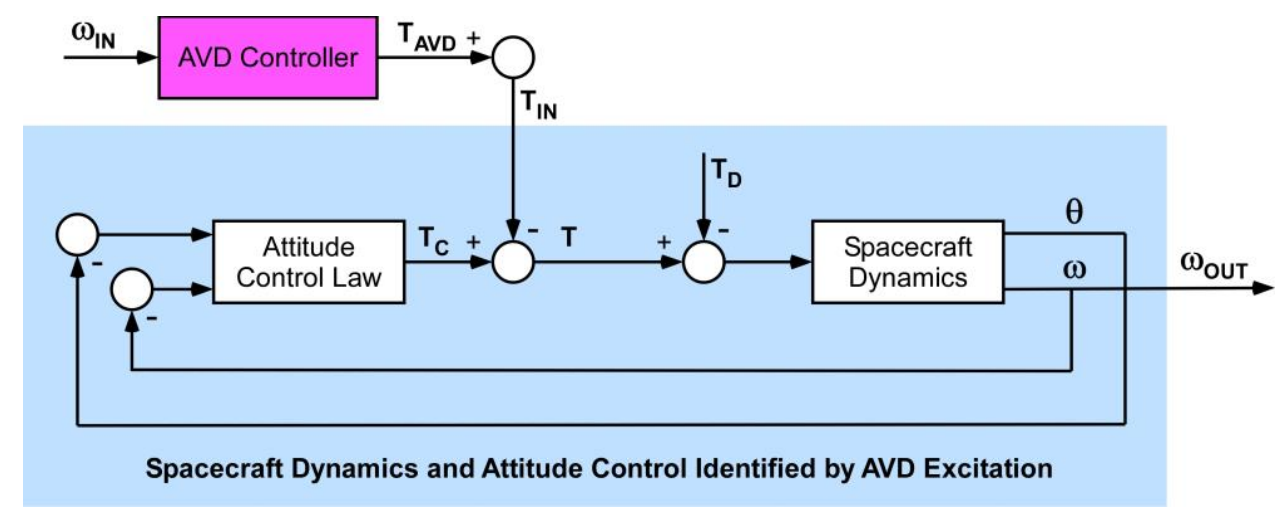

Figure 12. AVD Controller in Series with AVD Identified Model

Frequency domain transfer function Nichols plots from $\omega_{I N}$ to $\omega_{\text {OUT }}$ plotted as a magnitude response in decibels versus phase angle in degrees are shown in Figure 13. These figures are representative Nichols plots generated to characterize the GOES-16 AVD controller at all solar wing angles at $1^{\circ}$ increments for all three spacecraft body axes. Figure 13 shows the frequency domain characteristics of the AVD controller as it applies phase stabilizing gain at the GOES-16 appendage natural frequencies to quickly attenuate appendage vibration measured by the spacecraft IMU. Phase margins in degrees are measured along the zero decibel line between the transfer function modal peaks and the vertical lines at $360^{\circ}$ multiples of the $-180^{\circ}$ line. Phase margins are listed on each figure. Gain margins are the gain in decibels from the zero decibel line to the transfer function curves as they cross vertical lines at $360^{\circ}$ multiples of the $-180^{\circ}$ line. AVD controller minimum gain and phase margins at each solar wing angle at $1^{\circ}$ increments are evaluated and plotted versus the solar wing angle in Figure 14 for all three GOES-16 spacecraft body axes. The smallest gain margin is 11.5 decibels in the roll axis at a $41^{\circ}$ solar wing angle, and the smallest phase margin is $78^{\circ}$ also in the roll axis at a $219^{\circ}$ solar wing angle. The large stability margins for the GOES-16 AVD controller are another indication of the robustness of the vibration controller design. 

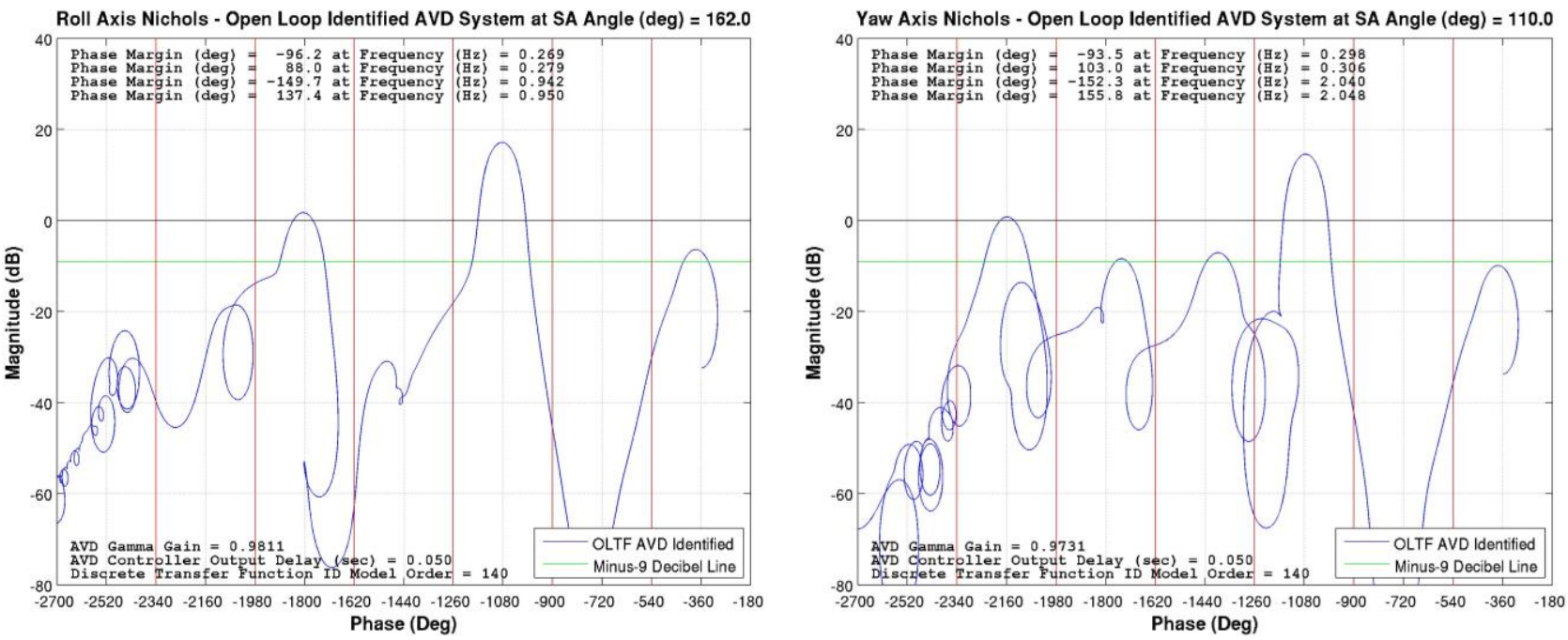

Figure 13. GOES-16 AVD Controller Stability via Roll and Yaw Axis Nichols Plots
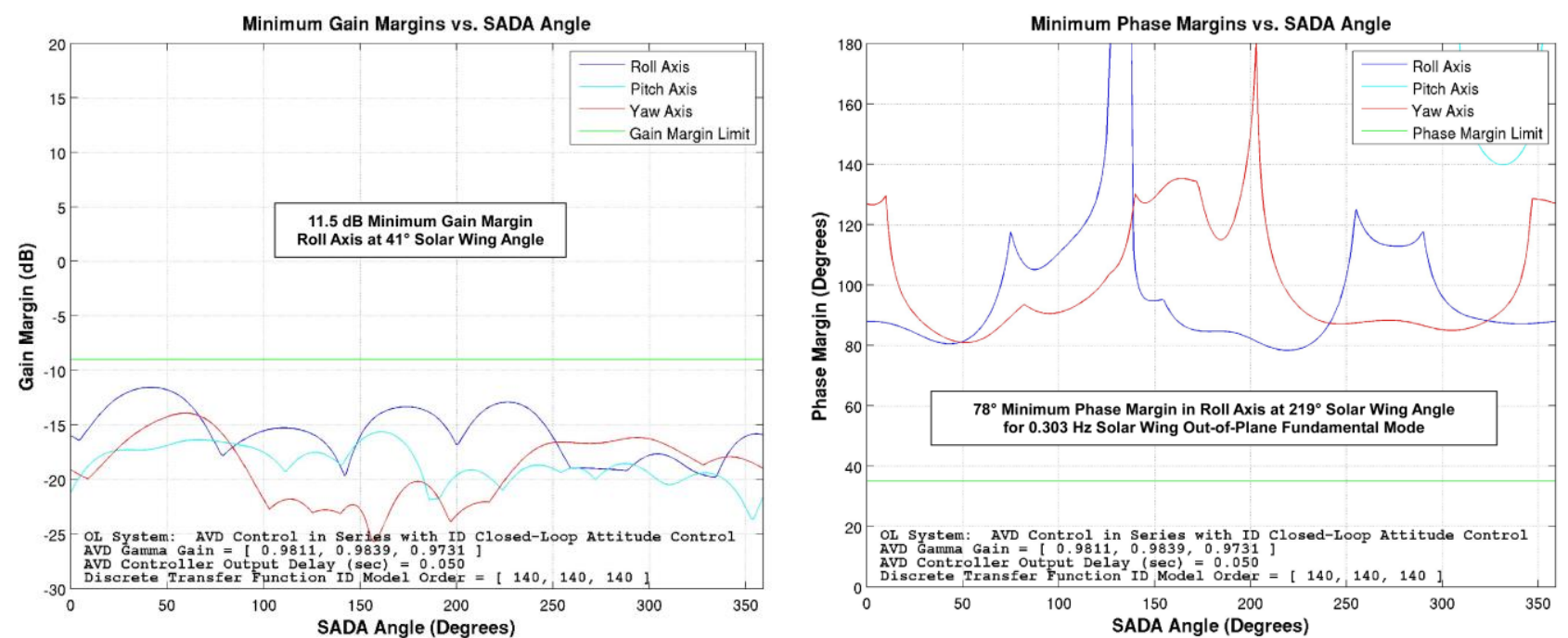

Figure 14. GOES-16 AVD Controller Minimum Gain and Phase Margins vs. SADA Angle

\subsection{GOES-16 Nadir Point Attitude Control System Bandwidth with AVD Disabled vs. AVD Enabled}

The AVD angular rate controller flattens the GOES-16 disturbance response curve as it provides additional disturbance rejection at the attitude control system bandwidth between $0.03-0.04 \mathrm{~Hz}$, as is evident in Figure 10 and Figure 11. This effect also influences the nadir point attitude control system bandwidth with the AVD controller enabled.

The bandwidth of the GOES-16 nadir point attitude controller is computed from the commanded attitude, $\theta_{C}$, to attitude response, $\theta$, transfer function as illustrated in the Figure 15 block diagram and written as Equation 8. This diagram divides the attitude controller into its constituent parts: the attitude control path, $G_{A}$, and the rate control path, $G_{R}$. The spacecraft dynamics is represented by the $D$ transfer function block. The familiar AVD identified Fourier model block diagram using the same convention is shown in Figure 16. The AVD identified model transfer function from disturbance torque input, $T_{I N}$, to angular rate response output, $\omega_{\text {OUT }}$, is written as Equation 9. Equation 10 shows 
that the transfer function from commanded attitude, $\theta_{C}$, to attitude response, $\theta$, can be constructed by augmenting the AVD identified model with the negative derivative of the attitude controller attitude path transfer function, $\left(-G_{A} / s\right)$. Therefore, the AVD identified model can be used to estimate the nadir point attitude control system bandwidth by augmenting it with an analytical expression for $\left(-G_{A} / s\right)$, as shown in block diagram form in Figure 17.

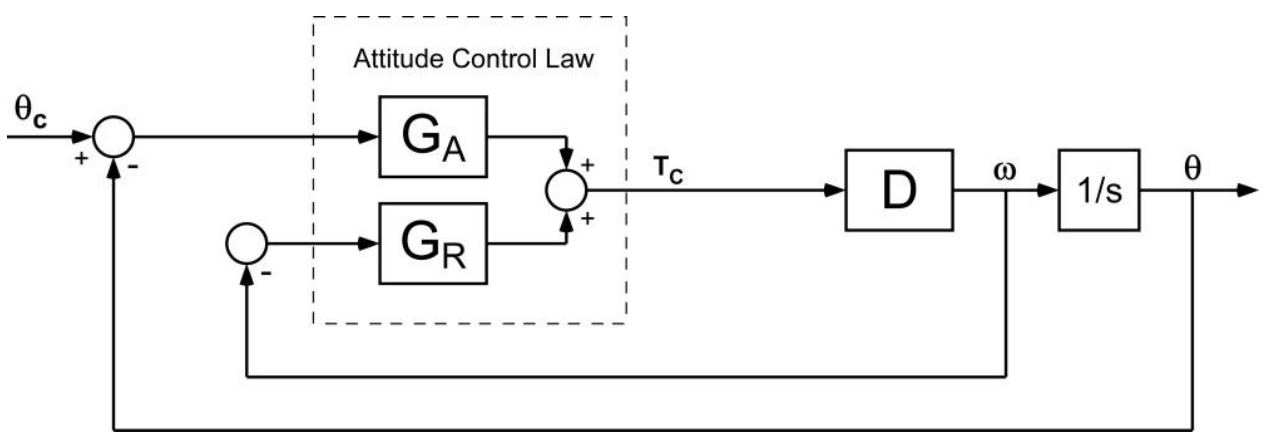

Figure 15. GOES-16 Nadir Point Attitude Control System Block Diagram

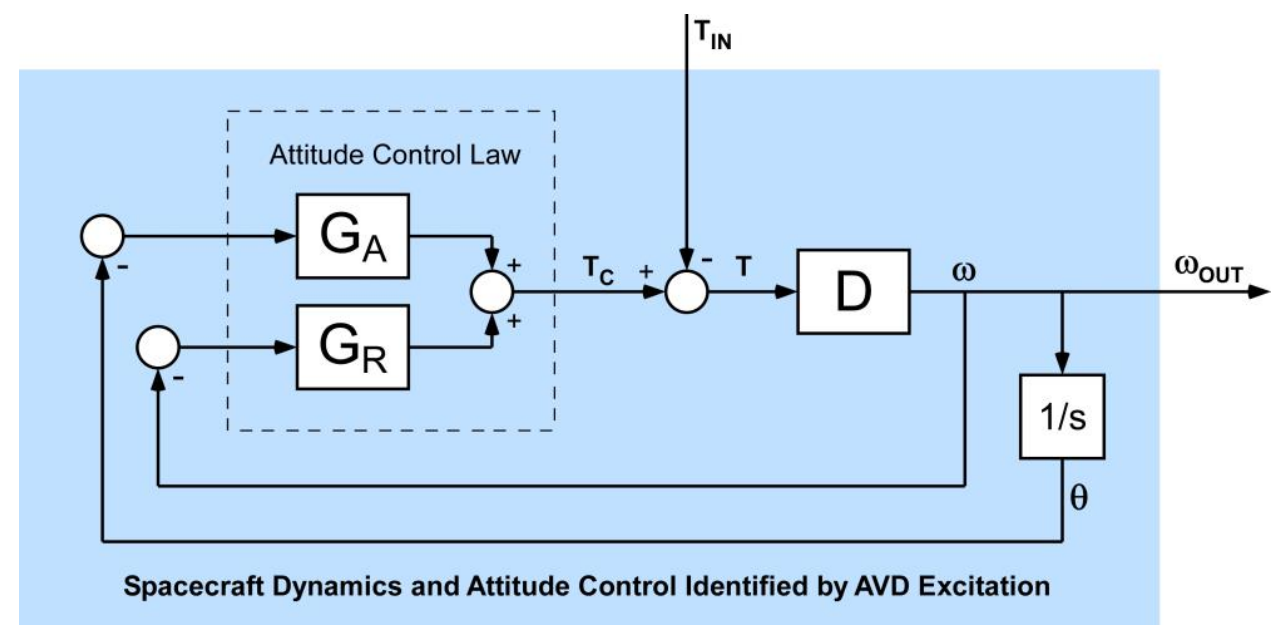

Figure 16. GOES-16 AVD Identified Fourier Model with Attitude Control Law Divided into Constituent Parts

$$
\begin{gathered}
\frac{\theta}{\theta_{C}}=\frac{D\left(\frac{G_{A}}{s}\right)}{\left[1+D\left(\frac{G_{A}}{s}\right)+D G_{R}\right]} \\
\frac{\omega_{O U T}}{T_{I N}}=\frac{-D}{\left[1+D\left(\frac{G_{A}}{s}\right)+D G_{R}\right]} \\
\frac{\theta}{\theta_{C}}=\frac{D\left(\frac{G_{A}}{s}\right)}{\left[1+D\left(\frac{G_{A}}{s}\right)+D G_{R}\right]}=\left(\frac{-G_{A}}{s}\right) \frac{-D}{\left[1+D\left(\frac{G_{A}}{s}\right)+D G_{R}\right]}=\left(\frac{-G_{A}}{s}\right) \frac{\omega_{O U T}}{T_{I N}}
\end{gathered}
$$




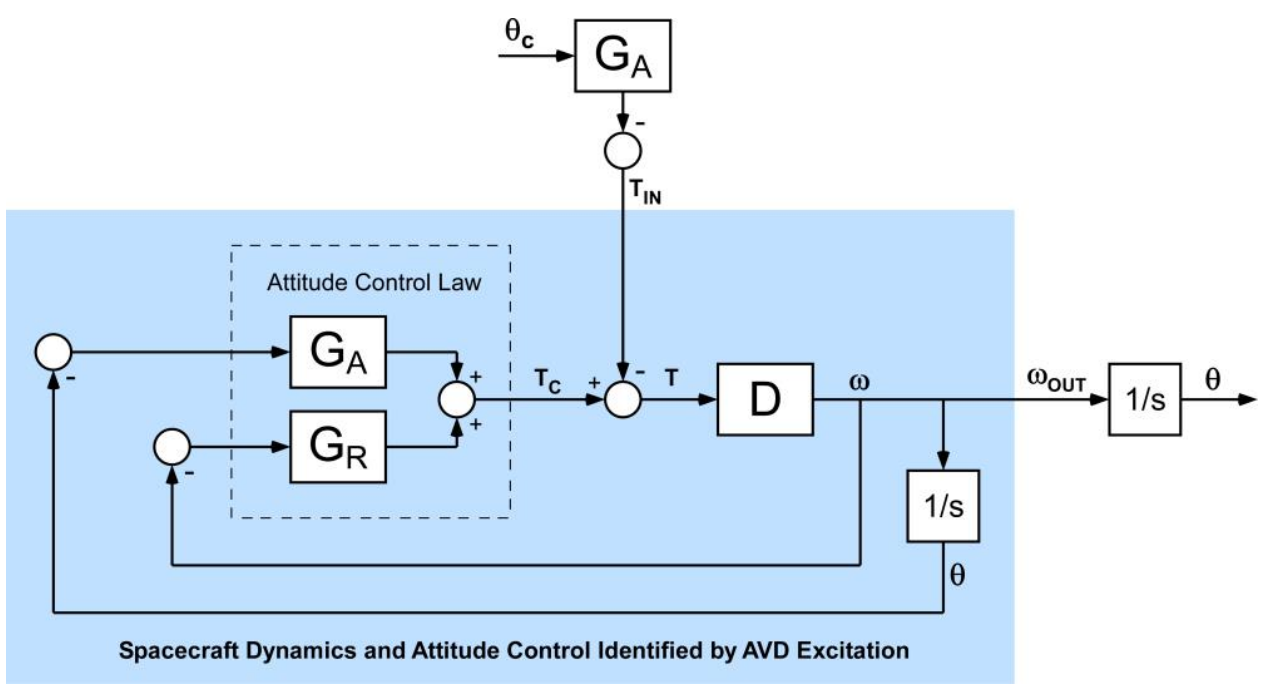

Figure 17. GOES-16 AVD Identified Model Augmented to Estimate Nadir Point Attitude Control System Bandwidth with AVD Compensation Disabled

Figure 17 is used to compute the commanded attitude, $\theta_{C}$, to attitude response, $\theta$, transfer function for the GOES-16 nadir point attitude control system with the AVD controller disabled, and Figure 18 is used to compute the same transfer function with the AVD controller enabled. Representative examples these transfer functions for GOES-16 are shown in Figure 19. The roll axis nadir point attitude control system bandwidth is $0.0444 \mathrm{~Hz}$ at the $-3 \mathrm{~dB}$ response amplitude for a $49^{\circ}$ solar wing angle with the AVD controller disabled; with the AVD controller enabled, the bandwidth drops to $0.02 \mathrm{~Hz}$. A similar effect is observed for the yaw axis at a $22^{\circ}$ solar wing angle in Figure 19. Because the GOES-16 primary mission operations concept is to stare at Earth while in nadir point attitude control mode, a drop in attitude control system bandwidth does not affect mission performance. The GOES-16 spacecraft is not required to quickly slew from one target to the next, which would require a high-bandwidth attitude control system.

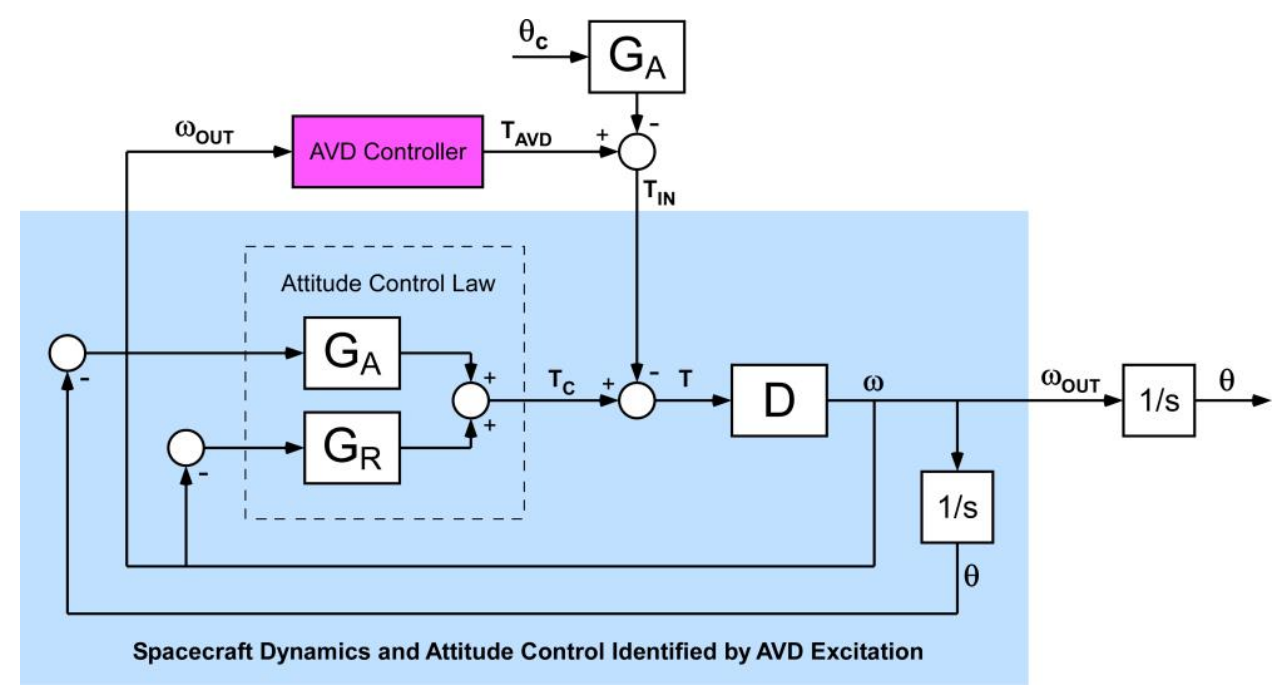

Figure 18. GOES-16 AVD Identified Model Augmented to Estimate Nadir Point Attitude Control System Bandwidth with AVD Compensation Enabled 

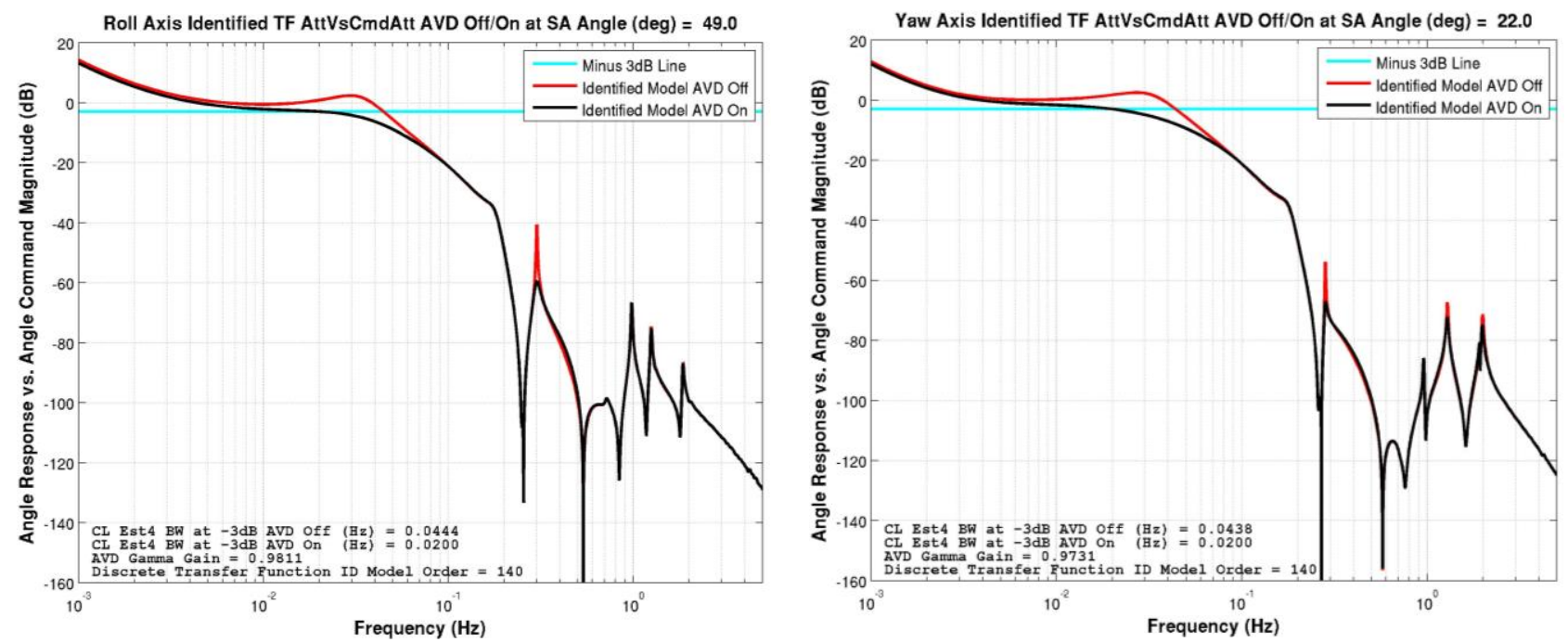

Figure 19. GOES-16 Nadir Point Attitude Control System Bandwidth with AVD Compensation Disabled and Enabled for Roll and Yaw Axes

Enabling the AVD controller always causes a drop in nadir point attitude control system bandwidth compared with the attitude control system bandwidth when AVD is disabled. Augmenting the AVD identified model with the $\left(-G_{A} / s\right)$ transfer function effectively rotates the GOES-16 disturbance rejection curves (Figure 10 and Figure 11) clockwise and applies gain to shift the rotated disturbance rejection curves up to unity magnitude. Therefore, as the AVD controller increases disturbance rejection at the attitude control system bandwidth (0.03-0.04 Hz), the AVD controller likewise causes a decrease in the nadir point attitude control system bandwidth, as Figure 19 shows. Also, because the AVD identified model angular rate disturbance rejection curves do not follow the typical -20 decibels per decade low-frequency roll-off, the attitude command to attitude response transfer functions in Figure 19 are not flat in the low frequency portion of the spectrum.

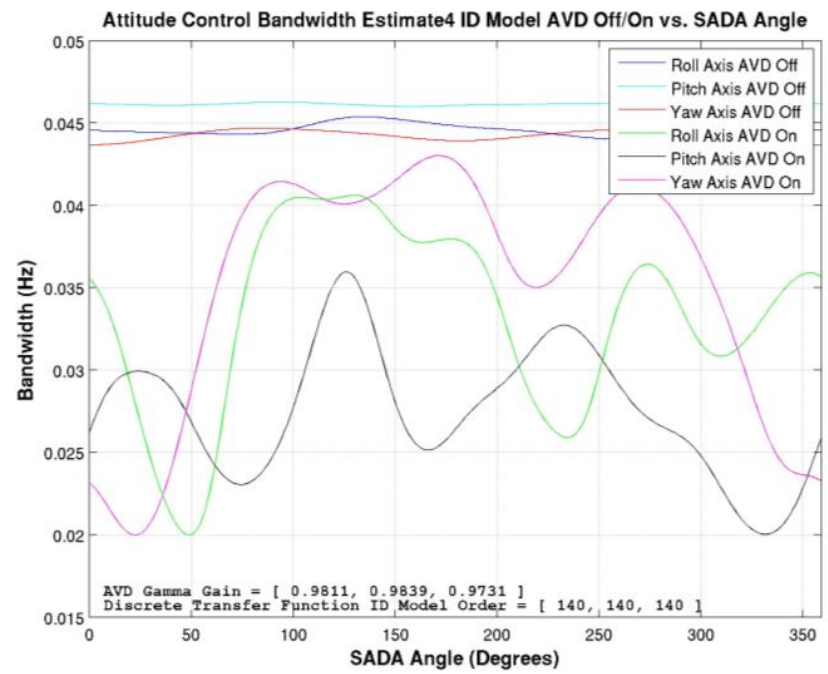

Figure 20. GOES-16 Nadir Point Attitude Control System Bandwidth with AVD Compensation Disabled and Enabled for All Solar Wing Angles 
Using the method described in this section, the GOES-16 spacecraft nadir point attitude control system bandwidth over all solar wing angles at $1^{\circ}$ increments with the AVD controller disabled and enabled is shown in Figure 20. The on-orbit nadir point attitude control system bandwidth with AVD disabled is approximately $0.045 \mathrm{~Hz}$ for all spacecraft body axes at all solar wing angles, whereas with AVD compensation enabled, the attitude control system bandwidth varies widely with solar wing angle. The AVD controller gains were selected to ensure that the nadir point attitude control system bandwidth is always greater than or equal to $0.02 \mathrm{~Hz}$ for all GOES-16 spacecraft body axes over all solar wing angles.

\subsection{GOES-16 Simulated Time Domain Impulse Responses with AVD Disabled vs. AVD Enabled}

Much of the analysis presented thus far was performed in the frequency domain using the frequency domain transfer functions for the GOES-16 AVD identified model (Equation 1) and the GOES-16 AVD controller (Equation 6). This section presents time domain simulation results using the GOES16 AVD identified model (Equation 2) and the GOES-16 AVD controller (Equation 7). Time domain simulation from disturbance torque input to angular rate response output using these GOES-16 models follows the block diagram formats already presented when the AVD controller is disabled (Figure 7) or enabled (Figure 9).

The time domain simulation using the GOES-16 flight models is forced by 0.05 second duration torque impulse inputs of $1.0 \mathrm{Nm}$ amplitude for the roll and pitch axes and $0.5 \mathrm{Nm}$ amplitude in yaw. The impulses occur every 24 minutes (every $6^{\circ}$ of solar wing rotation), and the simulation time duration is one full day starting at a $0^{\circ}$ solar wing angle. As occurs for GOES-16 in flight, the AVD identified Fourier model and the AVD controller filter coefficients are updated at every $2^{\circ}$ of solar wing rotation. The simulated angular rate responses are shown in Figure 21 through Figure 23 for the roll, pitch, and yaw axes, respectively, with the AVD controller disabled and enabled. Each figure shows time responses for one full day along with expanded plots covering 124 minutes in duration. Overlaying the AVD enabled time responses on top of the AVD disabled responses illustrates the effectiveness of the AVD controller to quickly attenuate GOES-16 appendage vibrations over the simulated orbit.
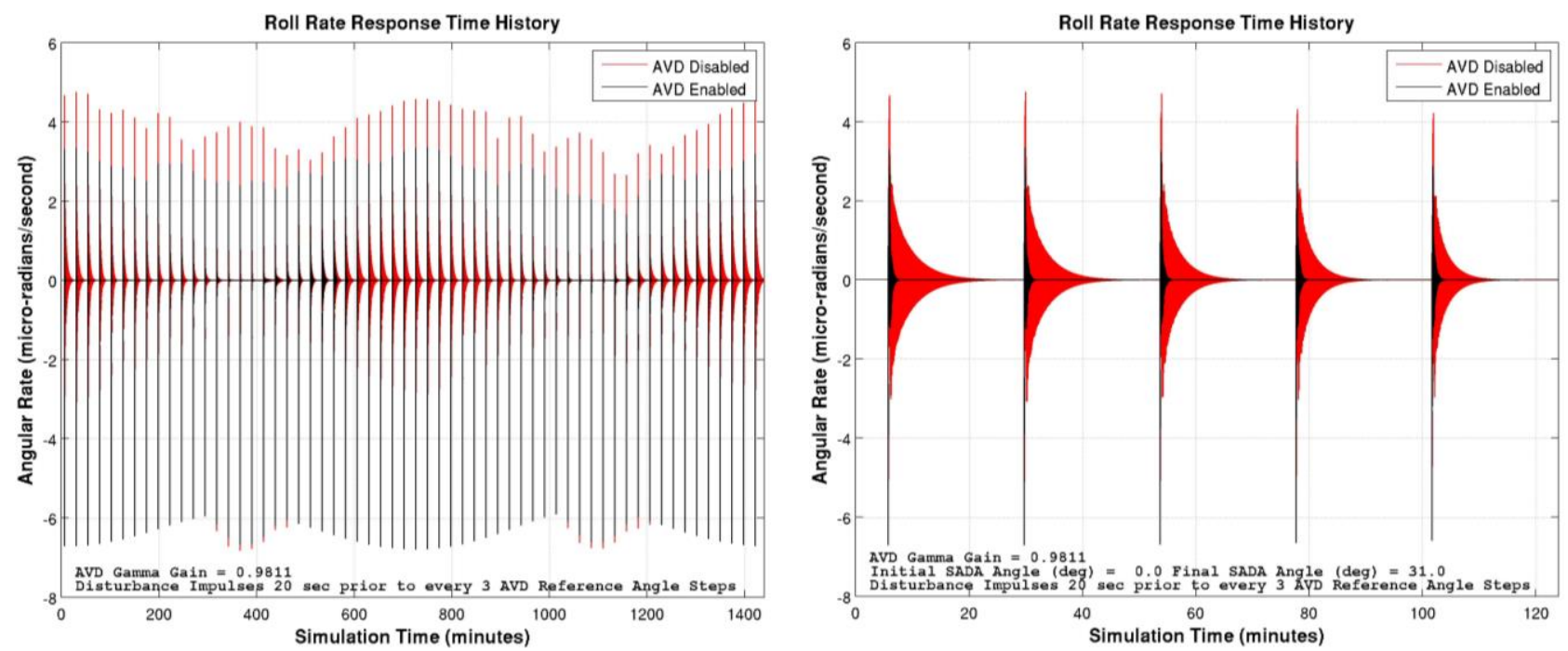

Figure 21. Simulated GOES-16 Roll Angular Rate Responses due to Torque Impulses 

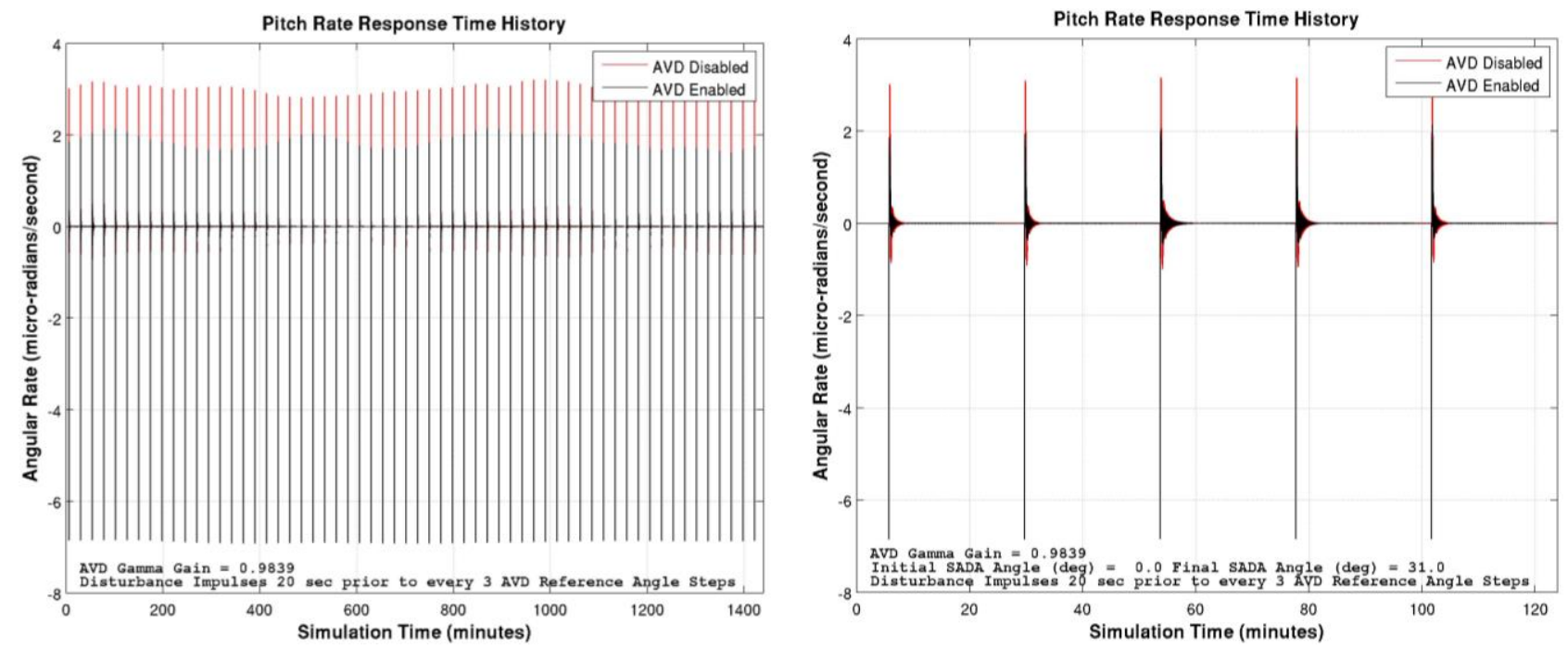

Figure 22. Simulated GOES-16 Pitch Angular Rate Responses due to Torque Impulses
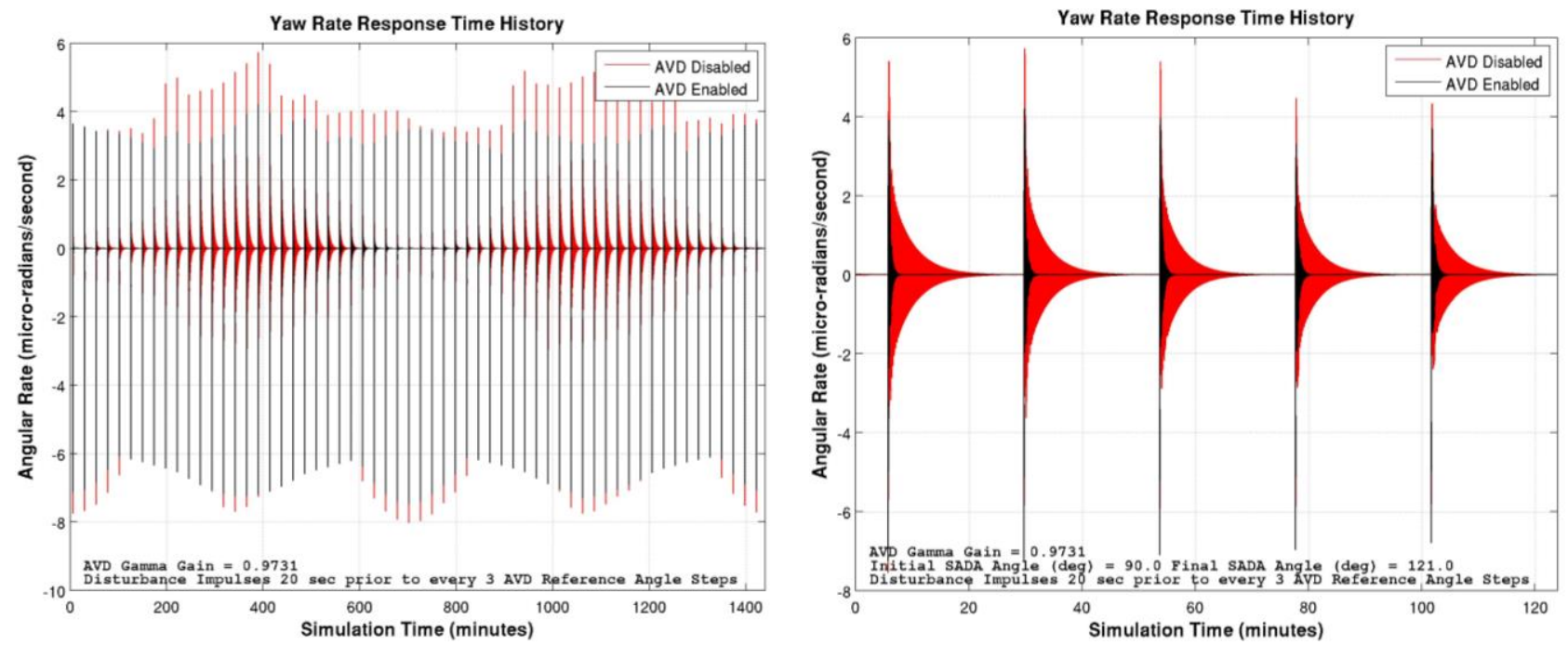

Figure 23. Simulated GOES-16 Yaw Angular Rate Responses due to Torque Impulses

Figure 21 and Figure 23 show that roll and yaw axis angular rate responses due to torque impulse inputs can last tens of minutes in duration with the AVD controller disabled, but the AVD controller attenuates the appendage vibrations in approximately one minute or less. The AVD controller is effective in reducing pitch axis appendage vibrations (Figure 22), but the pitch axis vibration attenuation is not as dramatic when compared with the roll and yaw axes. Perhaps the most important results illustrated by Figure 21 through Figure 23 are that GOES-16 angular rate time responses with the AVD controller enabled are always bounded over all solar wing angles, and appendage vibration decay times with the AVD controller enabled are always less than with the AVD controller disabled.

\subsection{GOES-16 On-Orbit Time Domain Disturbance Response with AVD Disabled vs. AVD Enabled}

GOES-16 on-orbit attitude error response is usually quiet and uneventful. However, every four days, arcjet thrusters fire for approximately one hour to perform north-south stationkeeping maneuvers, and low-thrust REA thrusters fire daily to unload reaction wheel momentum acquired during the previous 
24 hours. These thruster firing events excite GOES-16 appendage vibration and provide a good opportunity to observe AVD controller performance. On 29 January 2017 and 17 February 2017, temporally similar thruster firing events occurred. A north-south stationkeeping maneuver finished 30 minutes prior to the start of a momentum adjust maneuver. AVD compensation had not yet been enabled during the January 2017 event, but on the February 2017 occurrence, AVD compensation was enabled, which provided a means to compare GOES-16 appendage vibration responses as measured by the IMU. Figure 24 shows the GOES-16 roll axis attitude error response during these thruster firing events, and the pointing performance improvement with AVD compensation enabled is spectacular. Prior to activating AVD compensation, GOES-16 appendage vibration lasted tens of minutes after thruster firings, but with AVD compensation enabled, appendage vibration is quickly attenuated within one minute or less. The AVD algorithm is successfully operating on-board the GOES-16 spacecraft.

Roll Attitude Error GOES-16 North-South Stationkeeping \& Momentum Adjust

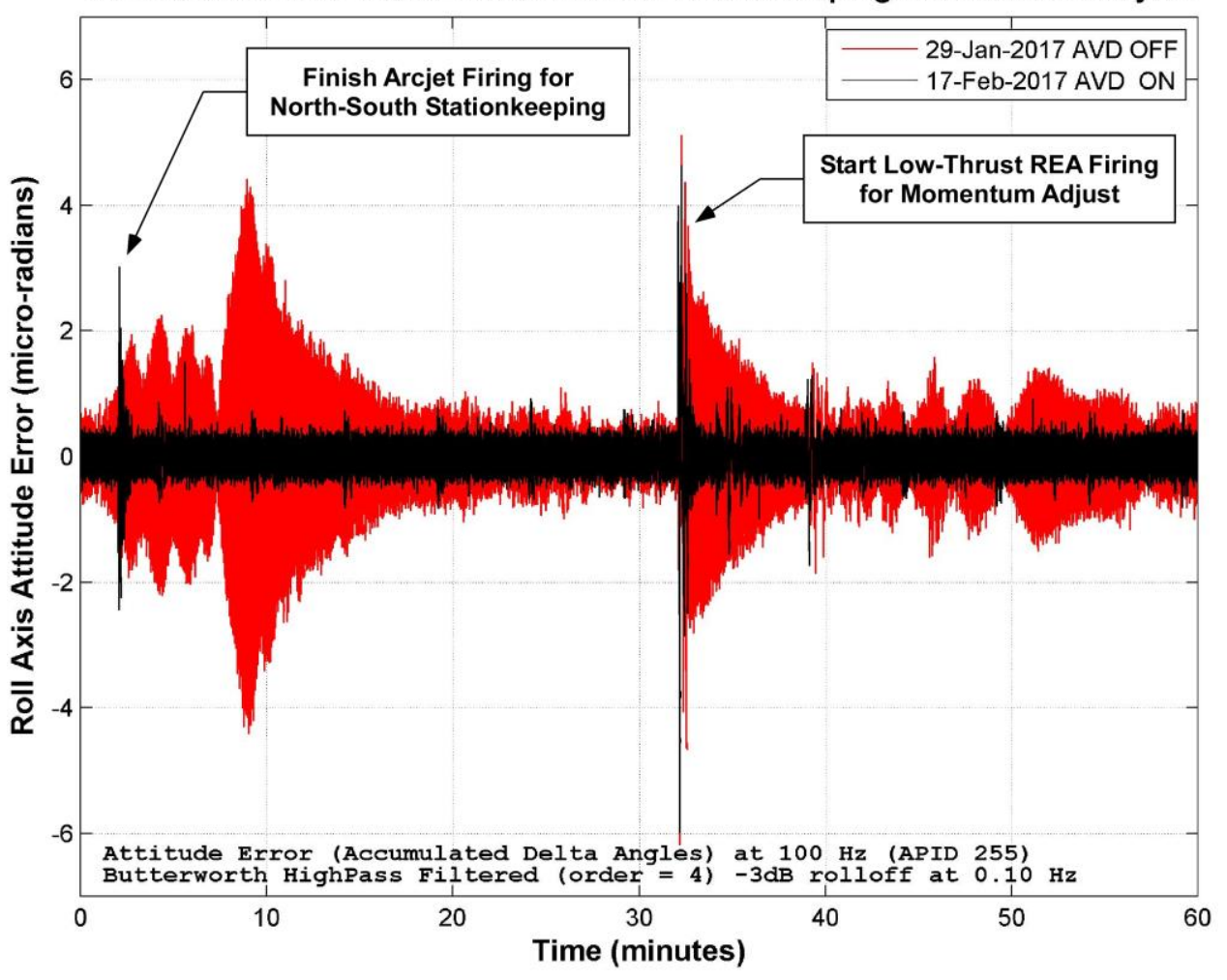

Figure 24. GOES-16 On-Orbit Disturbance Response during Thruster Firings with AVD Compensation Disabled and Enabled

\section{CONCLUSION}

The Active Vibration Damping controller designed for the GOES-16 spacecraft is operational after successful implementation on-board the GOES-16 flight computer. The AVD flight software algorithm is an innovative control system formulation, generating reaction wheel torque compensation commands from IMU input, which achieves more than a ten-fold increase in damping for the GOES-16 solar wing fundamental appendage mode. The AVD controller designed for the GOES-16 spacecraft is a system-identification based high-bandwidth angular rate controller which 


\section{GNC 2017 : 10th International ESA Conference on}

acts in parallel with the nadir point attitude controller. The AVD controller formulation is based not upon pre-launch simulation model estimates but upon on-orbit nadir point attitude control and timevarying spacecraft dynamics. AVD improves instrument pointing performance by applying robust phase stabilizing gain at the natural frequencies of the identified spacecraft appendage modes which modifies the spacecraft system dynamics, attenuates appendage vibration, and increases modal damping of the flexible appendages. AVD gain stabilizes all other spacecraft appendage modes with adequate margin to satisfy control system stability requirements. AVD provides additional lowfrequency disturbance attenuation control for all spacecraft body axes which improves the performance of the nadir point attitude control law while ensuring that the attitude control bandwidth does not fall below $0.02 \mathrm{~Hz}$.

\section{ACKNOWLEDGEMENT}

The authors wish to thank Alexander Krimchansky, Doug Freesland, Alan Reth, and Derrick Early at the NASA Goddard Space Flight Center and NOAA management for the opportunity to implement the Active Vibration Damping Controller on-board the GOES-16 spacecraft. The GOES-R series spacecraft are the first Lockheed Martin flight missions to implement AVD. This work was sponsored by the NASA Goddard Space Flight Center, Greenbelt, Maryland, under Contract NNG09HR00C.

\section{REFERENCE}

[1] Goodzeit, N.E. and Weigl, H.J., Active Vibration Damping (AVD) System for Precision Pointing Spacecraft, U.S. Patent: 20080272240, issued date November 6, 2008. 